Review paper

\title{
Instrumentation for diagnostics and control of laser-accelerated proton (ion) beams ${ }^{\text {th }}$
}

\author{
P.R. Bolton ${ }^{\mathrm{a}, *}$, M. Borghesi ${ }^{\mathrm{b}}$, C. Brenner ${ }^{\mathrm{c}}$, D.C. Carroll ${ }^{\mathrm{c}}$, C. De Martinis ${ }^{\mathrm{d}}$, F. Fiorini ${ }^{\mathrm{e}}$, \\ A. Flacco ${ }^{f}$, V. Floquet ${ }^{f}$, J. Fuchs ${ }^{\mathrm{f}}$, P. Gallegos ${ }^{\mathrm{g}}$, D. Giove ${ }^{\mathrm{d}}$, J.S. Green ${ }^{\mathrm{c}}$, S. Green ${ }^{\text {h }}$, B. Jones ${ }^{\mathrm{i}}$, \\ D. Kirby ${ }^{\text {h}}$, P. McKenna ${ }^{g}$, D. Neely ${ }^{\text {c}}$, F. Nuesslin ${ }^{j}$, R. Prasad ${ }^{\text {b }}$, S. Reinhardt ${ }^{\text {k}}$, M. Roth ${ }^{1}$, \\ U. Schramm $^{\mathrm{m}}$, G.G. Scott ${ }^{\mathrm{g}}$, S. Ter-Avetisyan ${ }^{\mathrm{b}}$, M. Tolley ${ }^{\mathrm{c}}$, G. Turchetti ${ }^{\text {n, J.J. Wilkens }}{ }^{\mathrm{o}}$
}

\footnotetext{
${ }^{a}$ Kansai Photon Science Institute, Japan Atomic Energy Agency, 8-1-7 Umemidai, Kizugawa, Kyoto 619-0215, Japan

b Centre for Plasma Physics, School of Mathematics and Physics, Queen's University Belfast, BT7 1NN, United Kingdom

c Central Laser Facility, STFC Rutherford Appleton Laboratory, Oxfordshire OX11 OQX, United Kingdom

' Dipartimento di Fisica, Universita di Milano and INFN Sezione di Milano, Milano, Italy

e Oxford Institute for Radiation Oncology (CRUK and MRC), University of Oxford, Oxford OX3 7DQ, United Kingdom

${ }^{\mathrm{f}}$ Laboratoire pour l'Utilisation des Lasers Intenses, Ecole Polytechnique, 91128 Palaiseau, France

g SUPA, Department of Physics, University of Strathclyde, Glasgow G4 ONG, United Kingdom

${ }^{\mathrm{h}}$ Department of Medical Physics, University Hospital Birmingham, Birmingham, B15 2TH, United Kingdom

${ }^{\mathrm{i}}$ Gray Institute for Radiation Oncology and Biology, University of Oxford, OX3 7DQ Oxford, United Kingdom

${ }^{j}$ Biomedical Physics, Klinikum Rechts der Isar, Technische Universität München, D-81675 München, Germany

${ }^{\mathrm{k}}$ Fakultät für Physik, Ludwig-Maximilians Universität München, 85748 Garching, Germany

${ }^{1}$ Institut fur Kernphysik, Technische Universitat Darmstadt, 64289 Darmstadt, Germany

${ }^{\mathrm{m}}$ Institute for Radiation Physics, Helmholtz-Zentrum Dresden-Rossendorf, 01314 Dresden, Germany

${ }^{\mathrm{n}}$ Departimento di Fisica, Universita di Bologna and INFN Sezione di Bologna, Bologna, Italy

o Department of Radiation Oncology, Technische Universitat Munchen, Klinikum rechts der Isar, D-81675 Munchen, Germany
}

\section{A R T I C L E I N F O}

\section{Article history:}

Received 28 February 2013

Received in revised form

5 September 2013

Accepted 7 September 2013

Available online 5 October 2013

\section{Keywords:}

Proton beam

Laser-acceleration

Dosimetry

Diagnostics

\begin{abstract}
A B S T R A C T
Suitable instrumentation for laser-accelerated proton (ion) beams is critical for development of integrated, laser-driven ion accelerator systems. Instrumentation aimed at beam diagnostics and control must be applied to the driving laser pulse, the laser-plasma that forms at the target and the emergent proton (ion) bunch in a correlated way to develop these novel accelerators. This report is a brief overview of established diagnostic techniques and new developments based on material presented at the first workshop on 'Instrumentation for Diagnostics and Control of Laser-accelerated Proton (Ion) Beams' in Abingdon, UK. It includes radiochromic film (RCF), image plates (IP), micro-channel plates (MCP), Thomson spectrometers, prompt inline scintillators, time and space-resolved interferometry (TASRI) and nuclear activation schemes. Repetition-rated instrumentati on requirements for target metrology are also addressed.
\end{abstract}

(c) 2014 The Authors. Published by Elsevier Ltd on behalf of Associazione Italiana di Fisica Medica. All rights reserved.

\section{Introduction}

Interest in laser driven proton (and ion) acceleration continues to be strong, with a potentially diverse range of applications. For medical applications the development of laser-accelerated proton [1] irradiation systems has been reported. The EURONNAC network (coordinated by CERN) has been set to explore potential crossbreeding between conventional and laser-acceleration. We note

is This is an open access article under the CC BY license (http://creativecommons. org/licenses/by/3.0/).

* Corresponding author. Tel.: +81 77471 3455; fax: +81 774713316

E-mail addresses: bolton.paul@jaea.go.jp, boltonpr@gmail.com (P.R. Bolton). that two funded ELI (Extreme Light Infrastructure) sites (in the Czech Republic and Romania) are using laser-accelerated protons as a strong feature of their infrastructures for science and applications. The Czech ELI site includes the "ELI-MED" structure that is devoted to exploring medical applications of laser-driven proton beams. Radioisotope production represents another potential medical application [2]. However, many fundamental significant challenges remain for development of laser-accelerated proton (ion) beams if they are to be applied to ion beam radiotherapy [3].

Many other applications are of significant interest such as highresolution proton radiography [4]. Applications to nuclear reactions, as probes for high-energy density states, and laser- 
accelerated proton sources are being considered in general [5]. For example, the potential for laser-driven high-brightness injectors in advanced accelerators (hybrid scheme for laser-driven accelerator system) is also considered [6]. Laser-accelerated protons can be applied to interrogations of sensitive material and generation of warm dense matter. Many of these applications will require welldiagnosed beams capable of controlled reliable delivery. We refer to the laser-driven facility as an integrated laser-driven ion accelerator system (ILDIAS). This review addresses ILDIAS instrumentation. It is not intended to cover all instrumentation but is based on material presented at the first workshop on 'Instrumentation for Diagnostics and Control of Laser-accelerated Proton (Ion) Beams' in Abingdon, UK. Understandably, medical applications requiring millimeter spatial accuracies may present the most stringent requirements [3]. For example, laser-driven ion beam radiotherapy (L-IBRT) will require stable, reproducible proton (ion) beams for deep-seated tumours at $250 \mathrm{MeV} / \mathrm{u}$ or more with an energy spread typically less than $1 \%$, dosimetry accuracy of a few percent (the legal requirement in many countries being less than $2 \%$ ), prompt noninvasive instrumentation, spot-scanning delivery to the tumour and demanding radiation protection controls [7]. Key enabling technologies must be developed to realise a laser-driven accelerator beamline. For source (laser plus target) development the importance of laser and target advancement is clear in laserplasma interaction studies. Energetic proton yields explore the laser-target parameter space. Beyond source studies, the development of suitable instrumentation and beam lines that can exploit the unique features of laser-accelerated proton bunches (notably the very large 'at source' angular divergence, broad spectral emission and high peak current) is critical and timely. Instrumentation enables monitoring and control of proton beam characteristics to understand, verify and optimise machine performance. We will ultimately need to correlate diagnostics of each laser pulse with those of the plasma and the emergent proton bunch that are uniquely attributed to each laser pulse.

We therefore require detection with single bunch resolution in space and time. Diagnostics must be implemented at the operational repetition-rate. Where possible, instrumentation should be noninvasive. However, laser-driven proton detection must be more robust than that of conventional ion accelerators due to the high peak current (high bunch charge of short duration) and the hostile detection environment near the source (laser target).

This report covers the following methods: radiochromic film and image plate detectors (Section 2), prompt inline diagnostics with beam profile scintillators and pixilated detectors (Section 3), Thomson spectrometers (Section 4), noninvasive correlated detection with time and space-resolved target interferometry (Section 5), diagnostics using nuclear activation (Section 6) and special 'targetry' diagnostic issues (Section 7).

\section{Ion detection with post-processing - radiochromic film and image plates}

Laser acceleration of ions (protons and heavy ions) is currently investigated using repetition-rated small and medium sized laser systems (pulse energy $\sim 0.1-10 \mathrm{~J}$ ) which operate at moderate repetition-rates and using larger systems at higher energy (pulse energy up to $500 \mathrm{~J}$ ) which are typically limited to a single shot operation mode. For a precise analysis of generated secondary particle beams it is mandatory, especially for the larger laser systems, to obtain complete information about the particle bunch conditions (the so-called phase space) following target irradiation by a single laser pulse. Of primary interest is the measurement of ion species, source size, divergence, transverse emittance as well as the spatial and energy resolved ion distribution. As radiochromic film and image plates are not sensitive to the ion species apart from the specific energy deposition, heavy ions can only be distinguished from protons by the $Z^{2}$ dependence of stopping power. Thus heavy ions are usually stopped within the first layers of detector stacks. Because the most powerful laser systems can generate a significant electromagnetic pulse, which can interfere with sensitive electronics sufficiently close to the experiment, film detection has been demonstrated as a valuable diagnostic because it is not sensitive to such electromagnetic noise. Electrons and X-rays on the other hand do expose the detectors, causing a dilute signal background. However, because of the very different energy deposition in consecutive layers, this contribution can be identified and accounted for.

\section{Radiochromic film}

In general, the energy deposited by particles penetrating one or more layers of the film detector is recorded and, precise calibration granted, the 2D image of the particle distribution can be obtained. If multiple films are stacked, each of them records the deposited energy and thus an energy resolved measurement (referred to as radiochromic film imaging spectroscopy) is possible [8]. Aspects of RCF calibration have been reported. For example, RCF has been calibrated with a microdensitometer for protons [9]. Information about differences in RCF analysis with different scanner types is available [9-11] and proton energy dependent dose correction factors in RCF have also been reported [12]. Protons of lower energies are stopped in the upstream layers, whereas protons with higher energies penetrate through and are stopped in films further downstream in the stack (Fig. 1). So, an energy bin, corresponding to an average proton energy, can be primarily attributed to each film. Although the fraction of the deposited energy of the penetrating high-energy protons can be minor (relative to the main Bragg-peak deposition) it still must be considered. The energy resolved proton distribution obtained from the film stack completes the beam reconstruction.

\section{Processing RCF data}

Radiochromic film (RCF) is a blue tinted plastic film (or yellow for EBT film), with a self-developing active layer which colourises upon exposure to ionising radiation. The active layer of RCF is made of an organic monomer which undergoes polymerisation upon irradiation. The polymer product exhibits significant optical absorption (darkens) and the colour is determined by the absorption spectrum of the polymer molecules. The degree of colouring is measured in terms of the film's optical density (OD): the reduction in the intensity of light that can be transmitted through the film. A crucial requirement of any film used for dosimetry is a known relation between the OD and the dose absorbed by the film. We define OD in terms of the fraction of light transmitted as follows:

$$
\mathrm{OD}=\log _{10}\left(I_{0} / I\right)
$$

where $I_{0}$ is the maximum light intensity without film, and $I$ the light intensity transmitted through exposed film [13]. When using a CCD scanner, the pixel value for a particular colour channel (or greyscale) is used as $I$. The relationship between OD and dose is usually near linear in the main range of operation. RCF can provide an accurate two-dimensional (2D) map of absorbed dose, with sub-mm spatial resolution and, depending on the film type, possibly down to the microns level. The radiochromic medium, in appropriate quantities and forms, can be used for a wide dose range, typically from $10^{-3} \mathrm{~Gy}$ up to $10^{4} \mathrm{~Gy}$ and this makes it attractive for many practical areas of radiation dosimetry.

For RCF that turns blue upon irradiation, the active layer has an absorption peak in the red region of the visible spectrum. By 


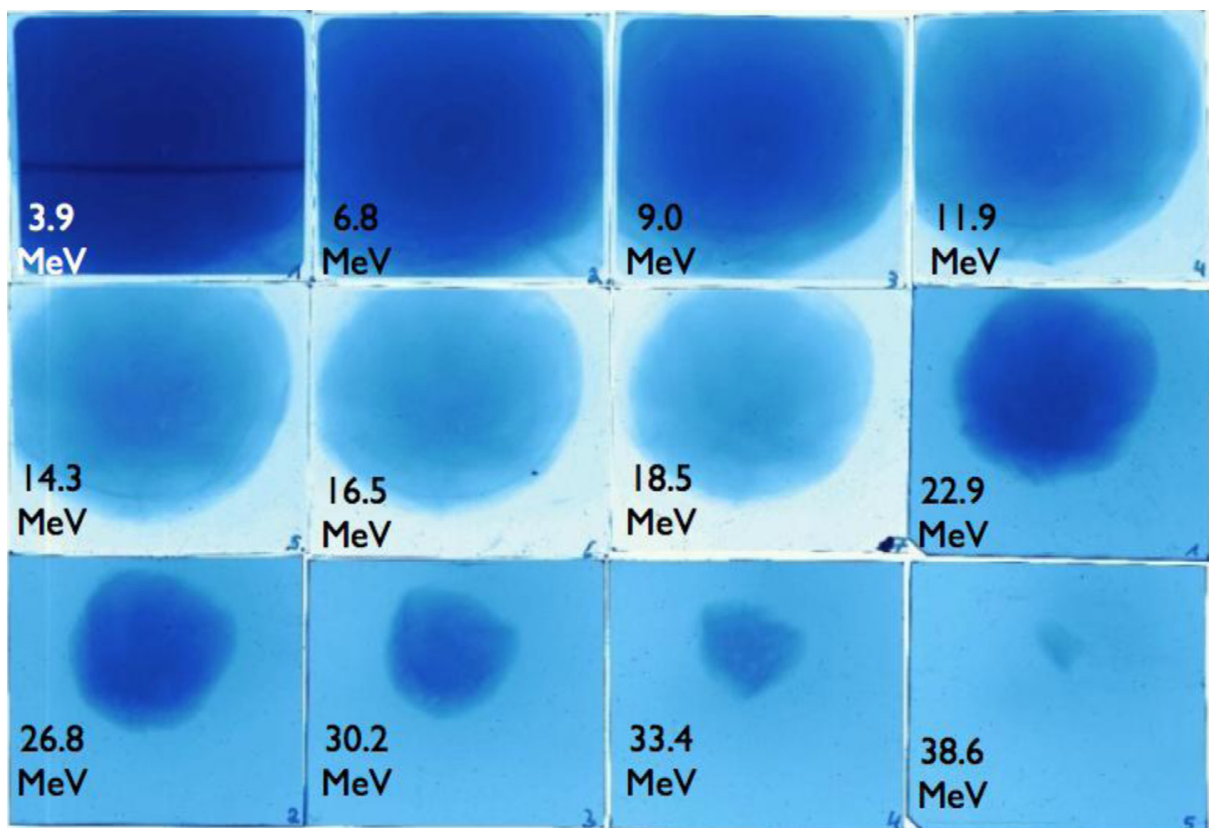

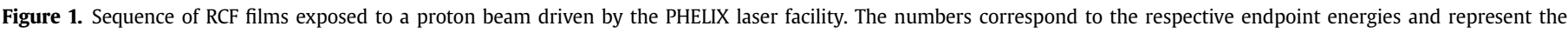
deposited dose mainly caused by the prominent energy deposition at the end of the particle range (Bragg-peak).

analysing the film with red light, it is therefore possible to increase the sensitivity of the measurement. The highest OD change per unit dose can be measured by using monochromatic light at the absorption peak wavelength, and it is possible to alter the dynamic range of the film by carefully selecting other visible wavelengths or using the green and blue channels of a white light scanner. The former method normally requires a scanning densitometer which is considered to be a slow and expensive method of digitising the film. However, cheaper commercial charge-coupled device (CCD) scanners have proved to be adequate if careful considerations are made. Many variables, such as temperature, UV light exposure, nonuniform light fields and polarisation effects can all have significant negative effects on the reproducibility and accuracy of RCF analysis $[10,14]$. However with careful control and correction protocols, the performance of the most widely used type of RCF, GafChromic film, has been found to be sufficient not just for experimental dosimetry but for clinical verification of radiotherapy treatment. GafChromic film is available as HD-810, MD-V2-55 and EBT. Their nominal suitable dose ranges are $0.001-8$ Gy for EBT, 2-100 Gy for MD-V255 and 10-400 Gy for HD-810 [15]. The elemental constituents of each film are slightly different, which may have important effects for the calculation of proton energy loss (Table 1).

In the environment of mixed charged particles and photon irradiation fields, these dosimetry films measure radiation dose which for a similar particle fluence will be mainly dominated by the ions, because their stopping power is higher compared to other particles or radiation being generated during the experiment

Table 1

Nominal atomic abundances in the active layers of GafChromic film, based on data received from ISP and not from direct measurement. HD-810 and MD-V2-55 are combined because they have exactly the same composition.

\begin{tabular}{lllllllllll}
\hline GafChromic model & \multicolumn{1}{l}{ \% Atomic abundances } \\
\cline { 2 - 10 } & $\mathrm{C}$ & $\mathrm{H}$ & \multicolumn{1}{ll}{$\mathrm{O}$} & $\mathrm{N}$ & $\mathrm{Li}$ & $\mathrm{Cl}$ & $\mathrm{Na}$ & $\mathrm{S}$ & $\mathrm{Br}$ \\
\hline EBT & 28.22 & 56.89 & 5.61 & 5.68 & 1.66 & 1.51 & - & - & - \\
EBT2/EBT3 & 29.61 & 58.33 & 10.79 & 0.06 & 0.82 & 0.19 & 0.11 & 0.03 & 0.06 \\
HD-810/MD-V2-55 & 29.14 & 56.80 & 7.12 & 6.94 & - & - & - & - & - \\
\hline
\end{tabular}

(e.g. electrons, or X-rays). According to the manufacturer RCF has a spatial resolution of more than $10^{4}$ dots per inch (dpi) or less than $2.5 \mu \mathrm{m}$. Due to its thickness RCF (a few $100 \mu \mathrm{m}$ typically) has mostly been used for proton beams. The stopping power of heavier ions scales with $Z^{2}$ ( $Z$ is the ion charge). Therefore heavier ions at energies available today from laser sources are usually stopped in the first layer.

Nearly complete (90\%) film colourisation appears within milliseconds after irradiation. However, further incremental colourisation occurs during the next day or more and it cannot be neglected. Consequently, it is recommended to read in the films for OD determination no sooner than 2 days after exposure (we note that the manufacturer recommends a waiting time of only $24 \mathrm{~h}$ for EBT and EBT2 film).

However, some groups also have used the transmission film scanner Microtek ArtixScan 1800 f instead of a densitometer to scan larger films faster with the same accuracy. More information about differences in RCF analysis with different scanner types can be found in Refs. [9-11]. The scanner calibration has been done with a grey scale wedge to convert the raw data into optical density (OD). The films were scanned with a resolution of 500 dpi and a dynamic range of 16 bit grey scales. The same scan parameters are used for both the calibration and the experiment in order to have the same conditions. This scanner could read ODs up to 2.5 , the saturation value. Then conversion of OD into deposited proton energy requires a calibration of the RCF sensitive layer using accurately measured proton doses. Usually such calibration has been done by different groups using conventional proton accelerators. During the last few years the chemical composition and also the thickness of the RCF sensitive layer have been changed by the manufacturer. This means that ideally a separate calibration for each type and production code is necessary. In addition to the effect of the composition difference the data differ from scanner to scanner so that no universal calibration curve for the RCF exists.

\section{Radiochromic film imaging spectroscopy}

In many applications related to laser-accelerated protons it is mandatory to characterise both the spatial and spectral profiles of 
the proton beam. Stacking radiochromic (dosimetry) films enable this. This technique is sometimes referred to as radiochromic film imaging spectroscopy (RIS), and is described for example in F. Nürnberg et al. [8]. A variation of this approach involves the use of structured targets. With the help of microstructured target foils and film detectors in a stack configuration it is possible to specify more proton beam parameters, with energy resolution and high spatial resolution. Micro-corrugations on the foil rear surface generate beamlets in the laser-accelerated proton beam. These perturbations in momentum space of the protons are embedded in the expansion of the beam to a point where the divergence angles originating from different microgrooves are well separated from each other. This is observed as a contrast pattern or image in the RCF detector. This effect is called microfocusing [1618]. Therefore the proton beam maps the rear surface structure of the foil onto the film detector. Due to deviations of the mapped image in the detector from the ideal image of the inserted grooves in the target, information about the transverse emittance and the size of the source for proton emission can be extracted. The symmetry and the divergence can also be determined by the imprint in the film. Details about this technique can be found in Ref. [8].

From a stack of RCF it is possible to deconvolve the initial proton spectrum and also resolve angular distributions of proton energy. Signal due to extraneous radiation (i.e. X-rays and electrons) requires some method of distinction and exclusion which is usually done by measuring the residual signal beyond the range of the highest energy protons. Several methods of RCF spectroscopy have been published $[8,9,19,20]$ and the proton stopping powers for the various layers of RCF are often obtained using the SRIM program [21]. A proton spectrum measured with the TARANIS laser at Queens University Belfast is shown in Fig. 2.

\section{Maximising dynamic range of radiochromic film with novel scanning techniques}

With a limited number of shots available at leading high power laser facilities, it is important to maximise the amount of data extracted from each film. This section outlines a novel method of increasing the recoverable dynamic range of RCF, extracting useful data which was previously unobtainable. Here we define dynamic range as the difference between the highest and lowest flux that can be measured (above the noise level), maintaining a monotonic relationship.

As stated previously, when RCF is exposed to high dose, generally the polymerisation reaction turns the film darker. However it is

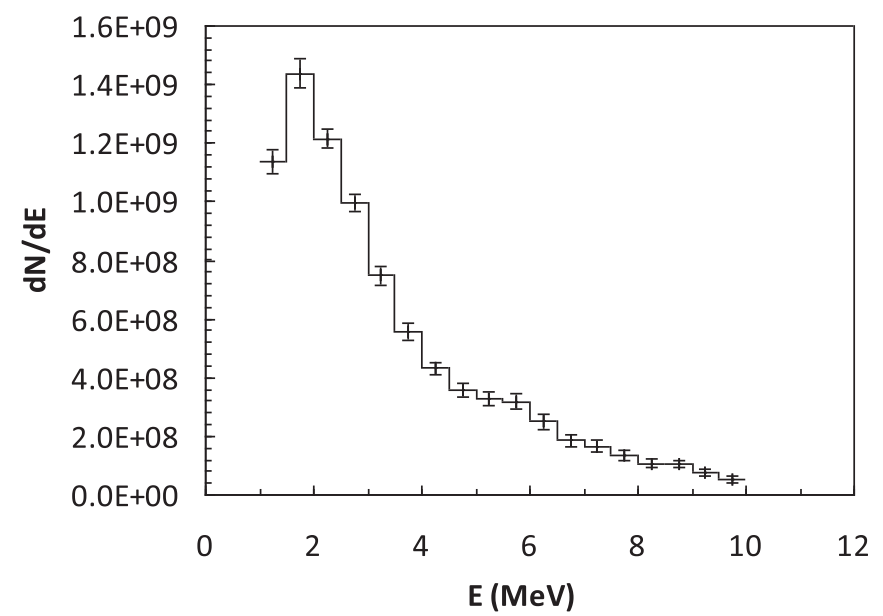

Figure 2. Proton spectrum from the TARANIS laser deconvolved from the depth-dose profile measured with an RCF stack. observed in practically every region of the spectrum that there exists a threshold above which the film's response is not a monotonic function of dose. Above this threshold dose the RCF optical density begins to decrease, making the film appear lighter in shade and so here a specific value of absorbed dose cannot be given using this technique.

Many laboratories use flat bed transmission scanners to analyse standard RCF. Here, pixel values of the scanned film can be calibrated to an individual scanner and converted to an optical density for each pixel. In this method care must be taken to ensure that a correction factor is introduced to account for any discrepancy between the optical density measured and a film's true optical density. This can be done using filters of known optical density. Flat bed scanners can also suffer from cross talk and this effect must be calibrated if small features are being examined. Calibration curves for RCF analysis using this method have been shown to be effective in the approximate range of $40 \mathrm{~Gy}$ to $2 \mathrm{kGy}$ when scanning in greyscale [8].

By adopting a multichannel approach to the analysis of higher sensitivity RCF it has been shown that the dynamic range of dose measurement can be increased [22]. Here we report findings with similar general trends for GafChromic HD-810 film. It should be noted that due to the inherently different sensitivities of the films we focus here on a dose range that is more than two orders of magnitude greater than the doses reported in Ref. [22].

In this technique the films are scanned in full colour and the images are post processed into their component red, green and blue channels. The three colour channels have different responses to dose as shown in Fig. 3 and this difference in response can be used to optimise and maximise the dynamic range of the film when scanned with a flat bed scanner. In Fig. 3 it can be seen that for the red channel a small increase in dose results in a larger increase in optical density, while becoming multi-valued at doses near 2000 Gy suiting the red channel to lower dose dosimetry. The blue channel is least sensitive and remains monotonic up to about $30 \mathrm{kGy}$, making this channel appropriate for more highly exposed film. The green channel behaves similarly to the greyscale and is suitable for use up to about $2 \mathrm{kGy}$, agreeing well with previous work [8]. When the three channels are combined they can be used to measure dose in the approximate range, $0.5 \mathrm{~Gy}$ to $30 \mathrm{kGy}$ when using HD-810 RCF.

In laser plasma experiments it is not unusual for the first RCF layers of a stack to be very highly exposed because they interact

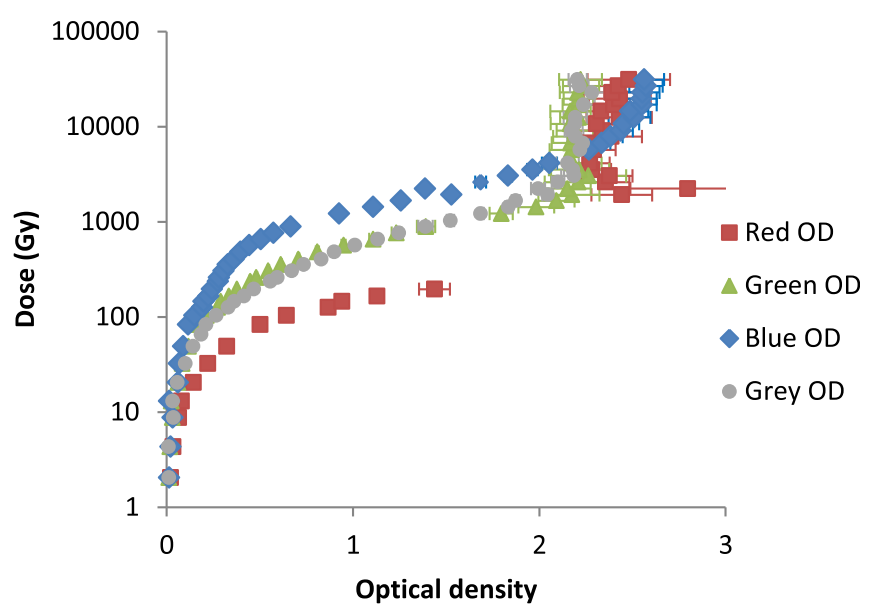

Figure 3. The optical response of GafChromic HD-810 radiochromic film when scanned using multiple colour channels and greyscale. 
with a large number of lower energy protons produced during such an interaction. Such films are commonly found to be above the flat bed scanner range even in the blue channel when using this threechannel technique. In an attempt to identify a region of the spectrum where it may be possible to recover dosimetric information, RCF was exposed to a controlled dose that was increased up to $200 \mathrm{kGy}$ using the cyclotron source at the University of Birmingham. By scanning the RCF films in a Shimadzu UV-1800 spectrophotometer a region of interest was found in the UV part of the spectrum around $300-350 \mathrm{~nm}$. Here the optical density scales well with dose across the full dose range up to and including $200 \mathrm{kGy}$ (Fig. 4 shows the curve for HD-810). The maximum measurable dose in the UV region may well exceed this value but we are unable to verify this. By analysing the spectrophotometer scans it is clear that in the UV we have identified a region of the spectrum where the dynamic range of RCF can be increased by almost an order of magnitude compared to that previously obtainable using the three colour method. These two methods may complement each other to afford dose measurement ranging from a few Grays to hundreds of kiloGrays.

Low energy limitation for radiochromic film

Reference has been made to the main limitation in the use of RCF for accurate proton dosimetry. It is the reduction in the response of the films for very low energy protons due to fundamental interactions of charged particles with matter. For high energy ions, the dominant interaction at almost all relevant energies from laser sources and for radiotherapy is Coulombic energy loss with atomic electrons. However, at low ion and proton energies, the higher linear energy transfer (LET) of these particles means that close to a particle track, the microscopic dose exceeds the dose range of the film. In essence, the polymerisation is locally saturated and so a portion of the energy deposited is not converted to a measurable signal. Relatively little attention has been paid to quantifying the energy dependence of the films to protons or ions until recently. The first attempt to measure an explicit dose-correction factor as a function of proton energy in the literature was by Piermattei et al. [23] examining MD-55. The measurements in Refs. [24,25] show an underresponse of MD-55 between 5 and 20\% for protons in the Bragg Peak region, depending on the initial energy of the beam. Kojima et al. [26] irradiated DM-1260 with a variety of ion species between 3 and $45 \mathrm{MeV} / \mathrm{u}$ and concluded that the higher the particle LET, the more significant the under-response. Martišíková and Jäkel [12] have found for EBT a large under-response of 25-35\% for carbon ions between 100 and $400 \mathrm{MeV} / \mathrm{u}$ and no under-response at all for protons down to $50 \mathrm{MeV}$.

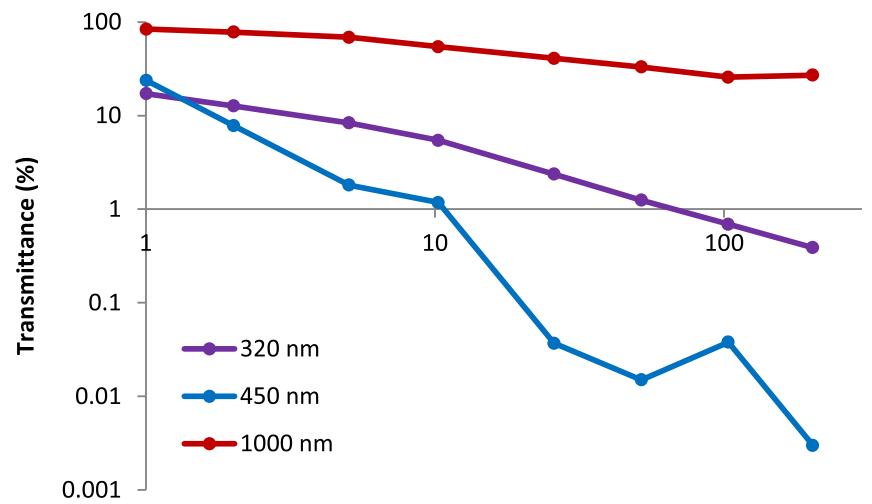

Dose (kGy)

Figure 4. Optical transmission of GafChromic HD-810 radiochromic film as a function of absorbed dose for multiple wavelengths.
For the proton energies encountered in currently available laser sources, this energy dependence is clearly of some importance. Kirby et al. [27] have made detailed measurements using a cyclotron-produced proton beam, and have characterised the change in response of MD-55 and EBT with proton energies. Their measurements reveal a number of important points: a) both MD-55 and EBT show a consistent "flat" energy response for protons above approximately $5 \mathrm{MeV}$ (EBT) and 8-10 MeV (MD-55), and b) below these energies, the response drops to around $60 \%$ of its higher energy value.

The consequences of this reduction in energy response have been explored in detail [20] for the low energy protons emitted in experiments at the TARANIS laser at Queens University Belfast. A correction methodology has been derived which allows a more accurate measure of the true proton spectrum to be recovered, resulting in significant changes especially in the low energy part of the spectrum, and increases in the estimated total proton yield by about 50\% [20].

\section{Image plate (IP)}

Image plate (IP) is made of phosphors with phosphorescent properties which can release the stored energy in a de-excitation (over a few hours). The IP is fabricated depositing BaFX:Eu2+ $(X=\mathrm{Cl}, \mathrm{Br}$ ) crystal grains on a polyester support film [28]. When photons or charged particles are incident on the IP, the electrons are promoted from Eu2 + to a meta-stable state where they remain trapped into the lattice defects. The energy stored in the IP can be retrieved by stimulating the excited meta-stable state. The stimulation can be done by photons, and then the energy is released as light and called photo-stimulated luminescence (PSL). The IP is read using a scanner. The surface of the IP is scanned by a laser diode that de-excites the meta-stable state generating UV light which is read by a photo-multiplier tube (PMT) and then converted into an electric signal. The output signal of the PMT is proportional to the light illuminating it. The signal is digitised by an analogue-to-digital converter $(A D C)$ and an algorithm in a 16 bit discrete number. Thus, this quantisation allows storage of the signal in a 16 bit format, in which each pixel represents a scanned position on the IP surface. The IP has very high dynamic range, high sensitivity to the stimulating radiation and its response is linear [29]. Once used, the IP can be erased by exposing it to intense light for at least $15 \mathrm{~min}$ and thus it can be used multiple times. Image plates can be used for detection of X-rays, neutrons and ions. However, because they must be scanned to retrieve the signal following exposure, they do not provide online measurements.

The properties of IPs (for example their sensitivity, dynamic range, detective quantum efficiency (DQE) and linearity of response) have been investigated over energy ranges relevant to medical applications [30-32], small-angle X-ray scattering experiments $[20,33]$ and neutron detection [34,35]. In the context of laser-plasma interaction experiments where broad energy spectra are typically observed, the response of IP to ions over a wide energy range is important. The response of the image plate detector to laser accelerated protons up to $20 \mathrm{MeV}$ is shown in Fig. 5 (left) [36]. The IP response to laser accelerated carbon ions with energies from $\sim 6 \mathrm{MeV}$ up to $40 \mathrm{MeV}$ has been measured [37] by cross-calibration with CR-39 [38] and is shown in Fig. 5 (right). The response to $\mathrm{C}^{6+}$ is maximum at $\sim 27 \mathrm{MeV}$.

\section{Prompt inline diagnostics}

For the last ten years the field of laser-accelerated ions has relied heavily on single use, passive imaging media such as radiochromic film (RCF), and CR-39 nuclear track detectors for high energy ion 

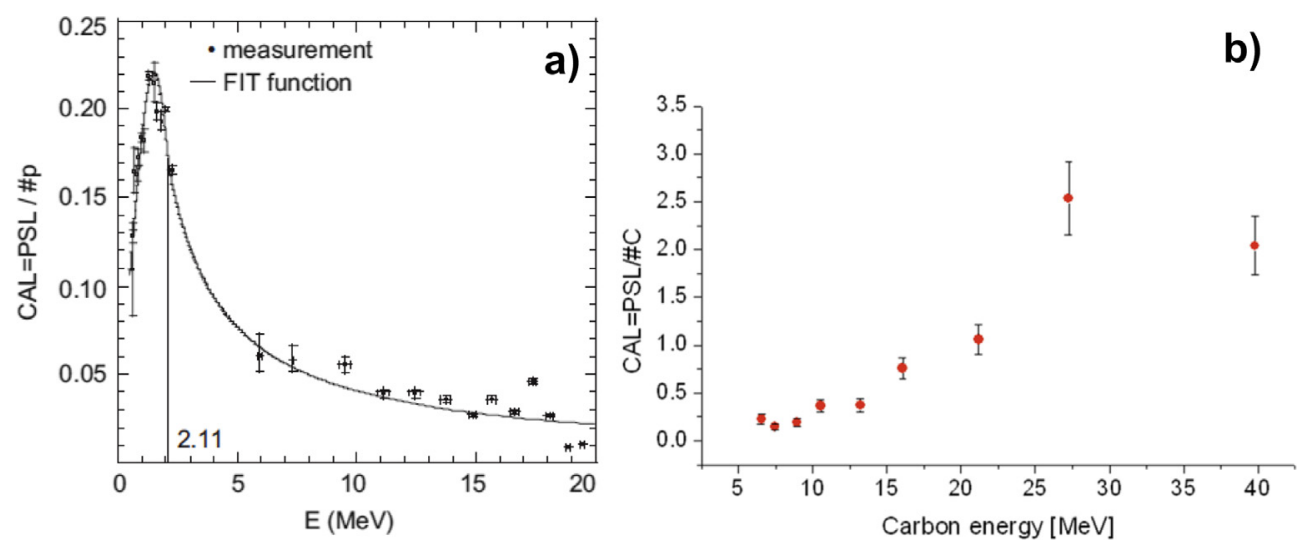

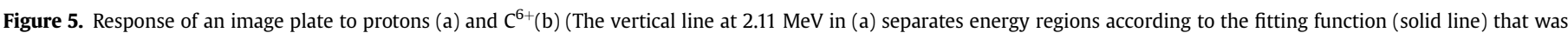

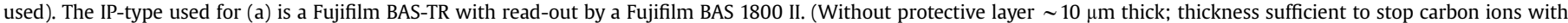
$\sim 800 \mathrm{keV} / \mathrm{amu}$ )

detection. However, the advent of ultra-intense, high repetitionrate laser facilities requires new techniques for in-situ ion detection capable of recording data for thousands of consecutive shots. Furthermore, for future radiotherapy with laser-accelerated ion beams, quantitative real-time detection is an essential prerequisite.

\section{Ion beam profiles using scintillators}

A scintillator-based diagnostic can spatially resolve the transverse profile of an ion beam over a number of specified energy ranges. By placing multiple sheets of organic (polyvinyltolouene based) scintillator in the beam path, the lowest energy ions are stopped in the first sheet, and higher energies are stopped in subsequent sheets. The collected optical signal is relayed from the interaction chamber, subsequently filtered into component wavelengths and imaged with a gated, intensified CCD camera (Fig. 6left). By choosing a set of scintillators that emit light over different wavelength regions, a single collection optic can be used to record transverse beam profiles for two or more ion energy ranges (Fig. 6right). The signals are separated just in front of the CCD by using one or two Fresnel biprisms in conjunction with two or four optical filters in order to produce multiple images, and hence beam profiles, on one CCD chip. The ability to closely monitor the beam pointing, flux and emittance for each proton (ion) bunch is essential if laser-driven ion sources are to be used for applications where shot-to-shot reproducibility is of critical importance.

A conventional RCF stack (see Section 2) offers higher spatial and spectral resolution, but re-useable plastic scintillators significantly increase the data acquisition rate while offering near-instantaneous single bunch feedback. However in order to become viable as an imaging medium, the scintillator light emission needs to be characterised over a range of proton energies and fluxes.

Results discussed here are based on tests with four scintillator types: BC-408 and BC-422Q from Saint-Gobain crystals and EJ-260 and EJ-264 from Eljen Technologies. The emission spectra of each scintillator were measured by illuminating a sample with a monoenergetic proton source where the light was recorded with an Ocean Optics spectrometer. Figure 7 shows the optical emission spectra. Overlap of emission wavelengths poses a problem for complete isolation of signals from each scintillator. To overcome this a combination of optical filters, inside the scintillator stack and in front of the CCD cameras, can be used to resolve blue, green and orange portions of the scintillation spectra (assuming a simple, three colour system). Hence three distinct beam profiles can be obtained, yielding valuable information about beam flux and divergence over three energy windows.

The scintillation process and subsequent optical transport must be characterised for absolute calibration of the proton beam profiler. With proper calibration, this diagnostic can potentially function as an in-situ proton calorimeter, measuring total beam flux over a range of proton energies. The optical response of each scintillator was tested using two monoenergetic proton sources; the University of Birmingham cyclotron and the Ion Beam Centre at the University of Surrey where $0.25 \mathrm{MeV}-28 \mathrm{MeV}$ protons were used. Figure 8 shows the proton energy dependent optical response for the BC-422Q plastic scintillator. The light output level was nonlinear for the energies tested, scaling as $E^{1.5} \pm 0.05$. Similar results were found for the other scintillators. However, care should be taken when analysing lower energies $(<1 \mathrm{MeV})$ where the scaling is less clear.
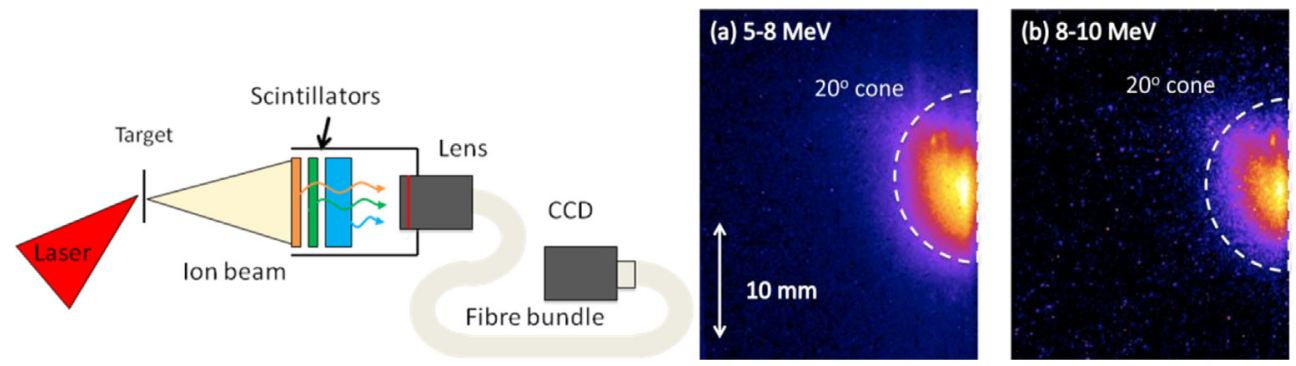

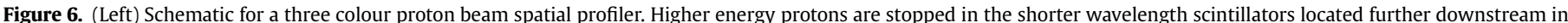

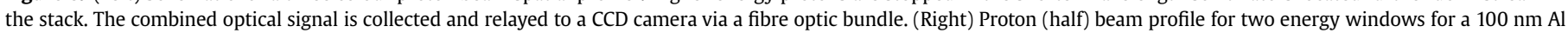
target irradiated at $\sim 5 \times 10^{20} \mathrm{~W} / \mathrm{cm}^{2}$ with high contrast $\left(>10^{9}\right)$. 


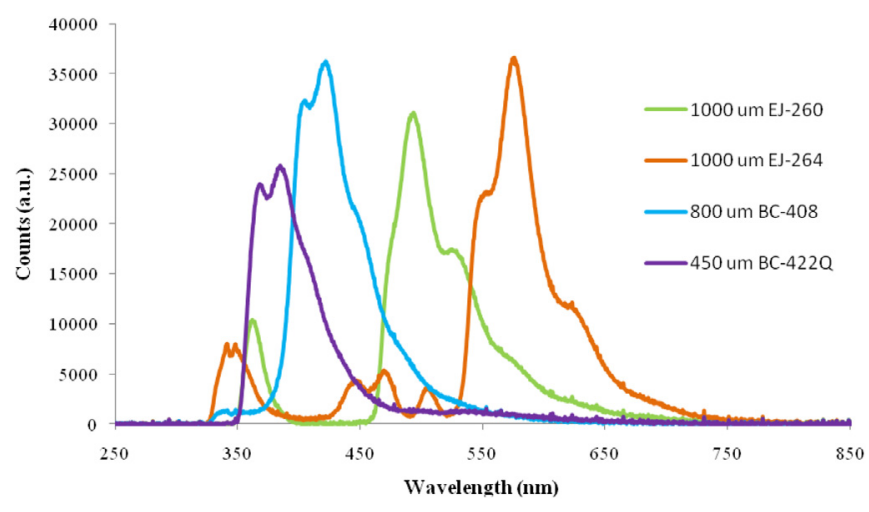

Figure 7. Emission spectra for EJ-260, EJ-264, BC-408 and BC-422Q organic scintillators.

Extensive work is still required to fully characterise the range of plastic scintillators currently available. More recently, detectors consisting of stacked BC418 sheets were tested where a spatial resolution of about $1.3 \mathrm{~mm}$ and spectral resolution near $1.5 \mathrm{MeV}$ were demonstrated [39]. In addition to extending the existing response to higher energies and heavier ions, the full dynamic range and spatial resolution of each scintillator need to be measured. While our initial tests suggest that light output scales linearly with proton flux, very high fluxes can lead to saturation and ultimately permanent damage. All of these issues must be addressed to clearly define the optimal operating parameters for a scintillator-based ion beam profiler.

\section{Pixel detectors}

Besides optical applications, pixel detectors are also widely used for X-ray and particle detection with sub-mm resolution. The principle of particle detection in a semiconductor detector is charge generation in the interaction between the incident particle and the sensitive detector volume [40]. The term pixel detector is used for a variety of different detectors, both monolithic and hybrid devices. The charge coupled device (CCD) [41] is the most common type of monolithic detector. Signal transfer to read-out electronics for further processing is accomplished by shifting the integrated charge, which is proportional to the deposited energy, from pixel to pixel. At the end of these shift registers, which use clocking frequencies of several $\mathrm{MHz}$, the signal is amplified in a single read-out node. Active pixel sensors, manufactured in a standard CMOS process where a first amplifier stage is already integrated into each pixel, achieve higher clocking frequencies and are even more radiation tolerant than CCD detectors.

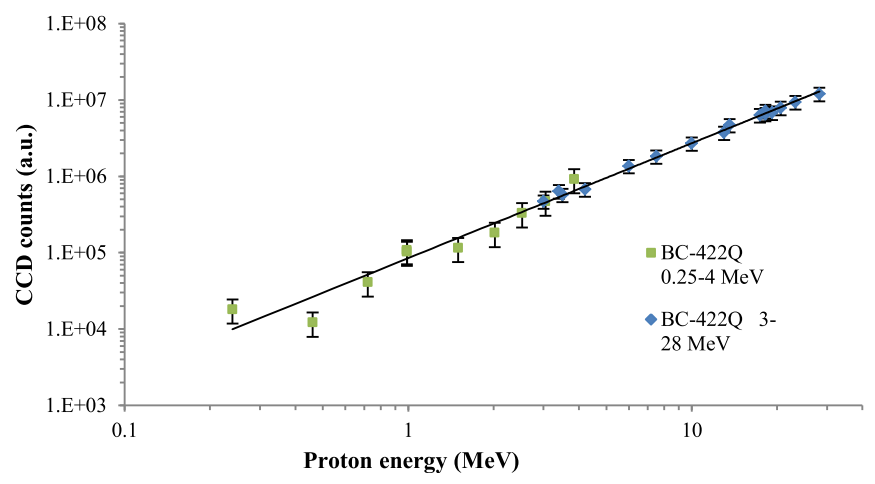

Figure 8. Scintillator response as a function of proton energy for BC-422Q.
There are high demands on the accuracy of biomedical beam monitors, typically better than $95 \%$. A typical treatment fraction, consisting of $10^{7}-10^{9}$ particles $/ \mathrm{cm}^{2}$, delivered in a few hundred $\mathrm{ms}$ in present proton therapy, could be supplied from a single laser shot, where the bunch duration is of nanosecond order. Therefore, for laser-driven accelerators, dose rates can be several orders of magnitude higher than those conventionally used today [42]. This can be problematic for detectors typically used in particle therapy such as multiwire ionisation chambers. The pixilated detector advantage is attributed to the large number of small single pixels, where, each represents a small diode detector. This reduces the number of particles per pixel to a level that is within its dynamic range, and affords measurement of a 2D fluence distribution with high spatial resolution. First tests of commercial CCD-based pixel detector, conducted at a conventional electrostatic accelerator using 10 and $20 \mathrm{MeV}$ proton beams (dc, pulsed and single ions), show good linearity between integrated detector signal level and particle fluence, and also sufficient dynamic range for this application. Single ion detection is likewise possible for detection of up to $10^{7}$ protons $/ \mathrm{cm}^{2}$ (shown at proton energy of $20 \mathrm{MeV}$ [43]). Similar results were obtained for a CMOS photodiode array that was tested on equal terms [43].

Charge-injection devices (CID) have been used for charged particle detection in inertial-confinement fusion experiments, where particles are emitted within time structures similar to those for laser-accelerated ions [44]. However, these experiments were accomplished at flux levels several orders of magnitude lower than those expected for laser-driven bunches. A sufficient saturation level of CIDs for intense laser-driven particle pulses thus needs to be demonstrated even if their radiation hardness would be beneficial for this kind of application. Nevertheless, more studies with laser-driven ion beams are required, investigating the shot-to-shot response as well as performance in the electro-magnetic pulse (EMP) noise environment of a pulsed laser.

\section{Micro-channel plates (MCP)}

A micro-channel plate (MCP) is a high resolution, spatially resolved, high gain electron multiplier $[45,46]$. The electron multiplication can span over several decades, which makes this detector type suitable for low intensity signals. MCP based amplifiers give a prompt response, (fluorescence time of order several nanoseconds) and can be used with either a continuous or pulsed (gated) biasing.

An MCP (Fig. 9) is a matrix of thin (4-25 microns in diameter) glass capillaries fused together and sliced into thin plates with typical thicknesses from a few hundred microns to one millimeter. Each capillary in the plate is an independent electron multiplier. A primary particle or photon impacting a channel wall generates secondary electrons. While drifting in an electric field applied

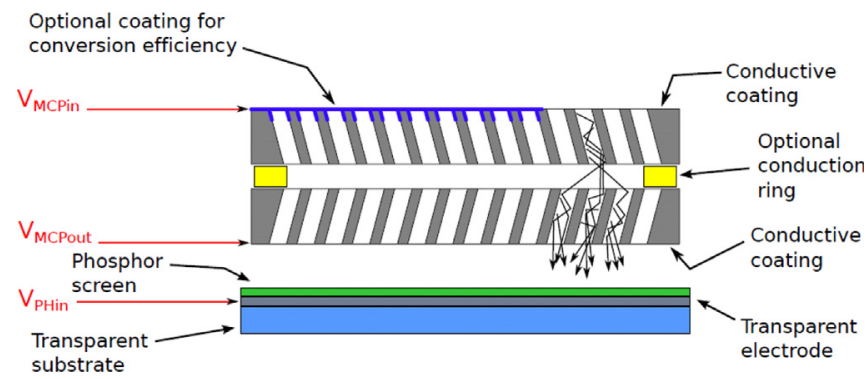

Figure 9. Schematic layout of an MCP Chevron stack, with two microchannel plates and a phosphor screen. Voltage signs and values might vary according to the chosen configuration, the phosphor type, the plate thickness and channel angle. 
between front and back sides the number of secondary electrons is amplified in subsequent collisions with the channel wall. The final electron charge distribution exiting the output surface drifts to a phosphor screen, where electrons are converted into photons that are collected by a camera system. The overall amplification in a single channel of one plate can be as high as $10^{4}$ and varies with the thickness of the plate, the angle between the channel axis and MCP surface (typically $4^{\circ}-12^{\circ}$ ), the value of the drift electric field and the channel coating material. Multiple MCPs can be stacked together to increase the total amplification to near $10^{7}$ (such as the Chevron stack in Fig. 9). The spatial resolution of a single plate is approximately equal to the capillary diameter. A second plate and drift distance between it and the phosphor screen introduce resolution losses (in a Chevron stack) An MCP is sensitive to ionising radiation (UV photons, electrons, ions and neutrons) which can be a problem with ion detection in laser ion acceleration experiments where an abundance of photons and electrons can saturate the detector. A magnetic spectrometer or Thomson parabola can be used to distinguish the desired ion signal from unwanted signals (Section 4).

Ion counting with an MCP requires adequate calibration. Many parameters, such as those typically produced in laser-driven accelerators, are known to affect the overall conversion efficiency (in photons per $\mathrm{MeV}$ per nucleon) for different ion species having a broad continuous spectrum. Calibration measurements can be performed with standard ion accelerators or in-situ in a laseracceleration experiment, using a single shot comparison of the MCP signal with that from a known calibrated detector (such as CR39) [47].

To calibrate a MCP-based detector in a laser driven ion acceleration experiment a slotted CR-39 detector can be placed in front of the MCP [47]. The counts per unit energy interval integrated across the width of the strip on the CCD image and the corresponding integrated number of protons on the CR-39 were measured. In order to correlate CCD counts to the number of protons, signals from two consecutive trace sections on the CCD were interpolated and then compared with the counts of the interleaved section of CR-39. Similarly, interpolation of two consecutive CR-39 trace sections and comparison with the interleaving CCD trace section was also done. In this way the ratio (counts/particle) as a function of proton energy was obtained. In the same manner the response for $\mathrm{C}^{6+}$ has been calculated. Figure 10 displays the response for protons up to $3 \mathrm{MeV}$ and for $\mathrm{C}^{6+}$ ions up to $16 \mathrm{MeV}$ per nucleon.

MCP-based ion detectors offer a very powerful solution for high sensitivity, real time detection of multiple ion species. The availability of plates with several centimeter diameters opens multiple possibilities for detecting and imaging the laser produced beams. Whenever multiple species are present in the analysed beam, the use of ad-hoc dispersion and selection devices (such as Thomson parabolas) is mandatory. More complicated solutions (see for example [46]) more fully exploit the surface of the detector plate for a deeper insight of the interaction products.

\section{Magnetic spectrometry and RCF comparison}

\section{Thomson spectrometer}

Spectroscopic diagnosis of laser-plasmas is well-established. The mass-spectrometer invented by J. J. Thomson in 1911 [48], has been widely used for analysing charged particles accelerated in laser plasma interactions. It simultaneously provides the distribution of accelerated particles as a function of their energy, momentum, and charge-to-mass ratio. The operation principle of the Thomson parabola spectrometer, which consists of the entrance pinhole, parallel electric $(E)$ and magnetic $(B)$ fields and a detector, is schematically shown in Fig. 11. In the small deflection approximation, the coordinates of the deflected charged particle with a given charge-to-mass ratio $\left(Z / M, M=A m_{\mathrm{p}}\right.$ the ion mass, $A$ the mass number and $m_{\mathrm{p}}$ the proton mass), passing through homogeneous electric and magnetic fields, on the detector plane located at a distance $D$ are:

$$
\begin{aligned}
& x=Z e \frac{E}{E_{i}} \frac{I D}{2} \\
& y=Z e \frac{B}{\sqrt{M E_{i}}} \frac{I D}{\sqrt{2}}
\end{aligned}
$$

where $E_{i}$ is the charged particle energy, $l$ and $D$ are the length of the electric and magnetic plates and its distance from the detector respectively (see Fig. 11a).

Ions with the same charge-to-mass ratio, $Z / M$ will trace the same parabola in the detection plane. The position of the particles along the trace depends on their energy, with higher energy particles closer to the so-called zero point. This is an origin point where the line joining the source and the spectrometer's entrance intercepts the detector plane, and where neutral particles and photons from the source would hit the detector. Equations (4.1) and (4.2) can be used to describe key spectral characteristics such as momentum per unit charge (with $B$ and $y^{-1}$ dependence), kinetic energy per unit charge (with $E$ and $x^{-1}$ dependence), ion velocity
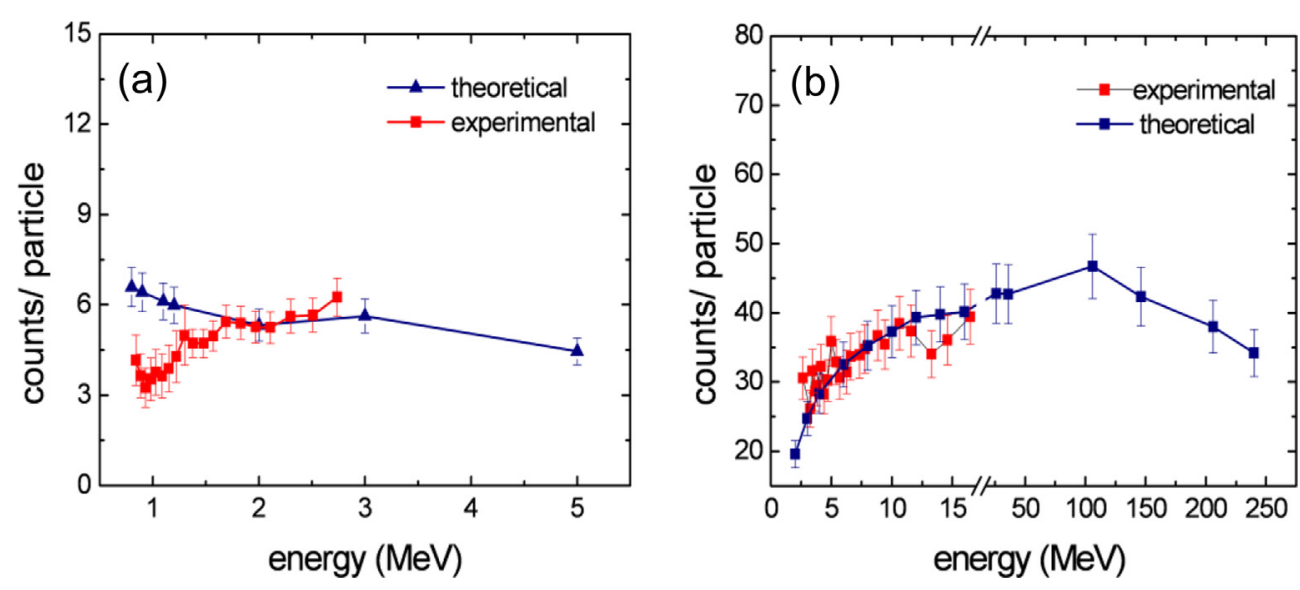

Figure 10. MCP detection efficiency measured with a laser-based particle accelerator; (a) protons; (b) $\mathrm{C}^{6+}$ ions. 
(a)

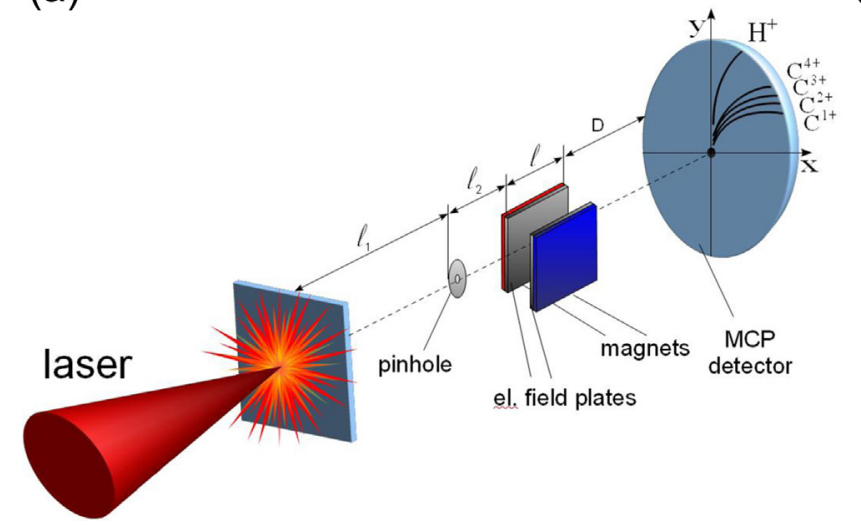

(b)

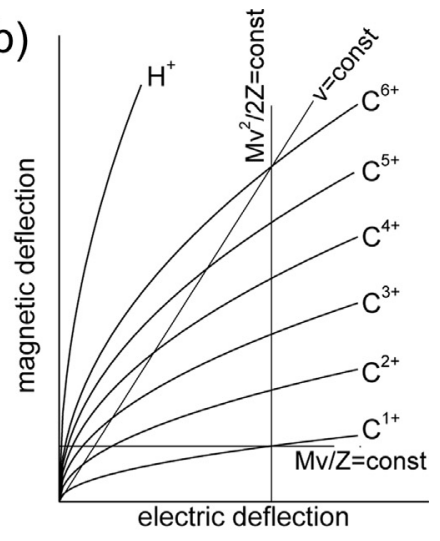

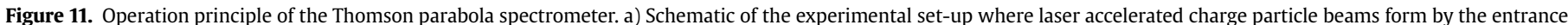

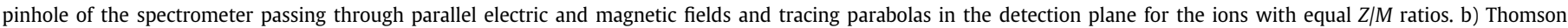
parabolas of C-ions and protons, and their intersection with a constant velocity line, a constant energy per charge line and constant momentum per charge line.

(with $E, B^{-1}, x^{-1}$ and $y$ dependence), and the charge-to-mass ratio (with $E, B^{-2}, x^{-1}$ and $y^{2}$ dependence).

Resolution along $x$ and $y$ axes depends mainly on the beam diameter set by the pinhole. Beam diameter places restrictions on the spectrometer capability to differentiate ion species and charge states. Spectrometer resolution depends inversely on the parabola width, $\delta$. Taking into account the laminarity of a laser-accelerated ion beam and the setup geometry, the width of the parabola is: $\delta=d\left(l_{1}+l_{2}\right) / l_{1}$, where $d$ is the pinhole diameter, $l_{1}$ and $l_{2}$ are the source-pinhole and pinhole-detector distances correspondingly.

Additional precautions are necessary for measuring ion beams produced from laser-plasma interactions. First, the entrance pinhole of diameter, $d$, can extract the hot electrons from the ion beam if it is smaller than the hot electron Debye radius $r_{\mathrm{D}}=\left(k T_{\mathrm{e}} / 4 \pi n_{\mathrm{e}} e^{2}\right)^{1 / 2} \approx 7.43 \times 10^{2} T_{\mathrm{e}}^{1 / 2} n_{\mathrm{e}}^{-1 / 2}(\mathrm{~cm}),\left(T_{\mathrm{e}}\right.$ is the electron temperature $(\mathrm{eV})$ and $n_{\mathrm{e}}$ is the electron concentration $\left(\mathrm{cm}^{-3}\right)$ ). For example, for $d=200 \mu \mathrm{m}$, electrons with a temperature above $T_{\mathrm{e}}>7.24 \times 10^{-10} n_{i} Z(\mathrm{eV})$ can be efficiently cut off, if the ion density is about $n_{i} \leq 10^{10} \mathrm{~cm}^{-3}$. Hence, due to partly removed electrons from the ion beam the former charge neutralised beam experiences a positive charge beyond the pinhole, which can cause ion beam broadening with a radius $r$ in the spectrometer during their propagation along the electric and magnetic field lengths, $l$. Broadening can be calculated as follows [49]

$$
\frac{\Delta r}{r} \approx \frac{e}{2 m_{\mathrm{p}}} \frac{l^{2}}{v^{3}} \sum_{j}\left(\frac{Z}{M}\right)_{j} I_{j}
$$

with $I_{\mathrm{j}}$ as $j$ th ion current density $\left(\mathrm{A} / \mathrm{cm}^{2}\right)$. If the ion density is high $\left(>10^{10} \mathrm{~cm}^{-3}\right.$ ) then the created volume charge can expand the beam.

Second, to prevent recombination of the ions on the way to the detector the pressure in the interaction chamber and spectrometers must not exceed about $10^{-5}$ mbar. Third, the fringe $E$ and $B$ fields in the spectrometer must be minimised. If spatial precautions have not been taken to adequately localise electric and the magnetic fields the field homogeneity can be compromised. However, to estimate the effective electric and magnetic fields all ion traces must be fitted such that they obey the same energy dispersion relation in the spectrometer. The $E$ and $B$ relations in the quadratic term of the parabolic traces can be used to determine the acting effective magnetic field if the electric field is known with good accuracy.
The spectrometer becomes uniquely valuable for quantitative analysis of laser-accelerated particles if detectors with single particle sensitivity are used, such as an absolutely calibrated multichannel-plate (MCP) coupled to a phosphor screen (see Section 3.3). If this is imaged onto a CCD camera chip, a relation can be established between registered counts on the camera image and the impact of a single ion onto the MCP. This affords parameter measurement of ion yields from a single laser shot with high dynamic range as well as spatial and spectral resolution. Such capability would be highly beneficial for understanding relevant plasma dynamics and particle acceleration mechanisms. Although standard Thomson spectrometers are very useful, in recent years several modifications have been introduced to allow a more comprehensive analysis of the ion acceleration phenomena. The construction of a Thomson spectrometer which employs microchannel-plate (MCP) detection (with single particle sensitivity) enables: temporally gated [47] and spatially resolved [50] detection of accelerated ions (at the detector plane); the simultaneous measurement of ion and electron spectra along the same observation direction [51]; precise measurement of the proton/ion trajectories and its applicability for proton deflectometry [52,53] and tomography of the ion source $[54,55]$. A Thomson spectrometer that combines MCP detection with a tunable magnetic field has also been developed [56]. Additionally, complementary ion and XUV spectrometers for laser-plasma diagnosis have been developed [57] for qualitative analysis of the ion emission and XUV spectra which can provide detailed information about the plasma conditions, and possible correlations between the energetic proton emission and the XUV plasma emission.

These charged particle diagnostics are not mutually exclusive and are often fielded simultaneously along different observation lines. They allow one to conduct more thorough research of relevant processes for a single laser shot. Simultaneous measurement of plasma parameters attributed to laser irradiation from a single pulse is an important requirement for observing the shot-to-shot fluctuations.

\section{Comparison with radiochromic film $(R C F)$ detection}

Spectral measurements performed with RCF stacks and the magnetic spectrometer (MS) can be complementary. With a known calibration, radiochromic film data from a stacked configuration can be deconvolved to determine the deposited proton energy for 
each film. With a fitting procedure, the incident proton spectrum can be deduced. This has been discussed in more detail in Section 2.1.1. However, unlike the MS, the RCF technique measures the whole beam angular distribution with a coarse energy resolution. Therefore only the RCF inferred spectrum is used to extract the total energy in the proton beam and the energy conversion efficiency for proton acceleration (laser pulse energy to full spectrum proton energy).

Radiochromic film reveals that proton beam divergence follows a universal curve $[58,59]$ when scaled to the beam maximum energy (see Fig. 12). This is because the relative spatial shape of the electron sheath that accelerates the proton beam is always the same in the frame of the TNSA acceleration [60,61].

In contrast to the discrete coarse results from an RCF stack, the energy spectrum measured with the MS is continuous. An MS provides information only at a particular angle (with an insignificant angular spread) that is set by spectrometer alignment relative to the ion source (laser target) making it therefore much more alignment sensitive than an RCF stack. Misalignment can mean missing the high energy region of the spectrum which can be preferentially directed orthogonal to the rear surface of the laser target with a minimum divergence. If the aperture of the spectrometer is well centered on the axis of the proton beam (nearly normal to the target rear surface), the spectra measured by the RCF stack and the MS (in units of $1 /\left(\mathrm{MeV}^{*} \mathrm{str}\right)$ ) can agree relatively well. In order to measure the two spectra simultaneously, the on-axis component of proton beam can reach the MS through an aperture at center of the RCF stack.

\section{Diagnostics correlated to the proton bunch - noninvasive laser technique to determine characteristics of hot electrons that accelerate the ion beam}

To understand the underlying ion acceleration mechanisms, it is essential to accurately characterise the resulting proton beam and the hot electrons generated at the acceleration source. The measurements desired are their spectrum, characterised mainly by their mean energy or "temperature" (usually a quasi-Maxwellian distribution), and their number or density at the target rear surface (acceleration location).

One way to retrieve the sheath temperature of the electrons which accelerate protons along the normal axis is to consider the slope of the proton spectrum. A fit to this slope estimates the sheath temperature. This fit also gives the total hot electron number within the sheath $N_{\text {hot }}$ [62] which contributes to proton acceleration. This

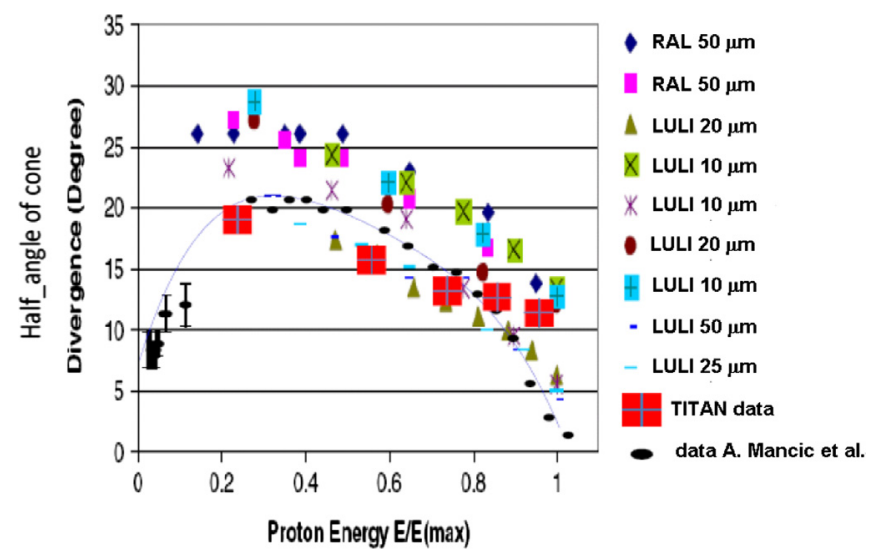

Figure 12. Compiled measurement of proton beam divergence obtained from several laser facilities (see text for references). is obtained from the model of P. Mora $[63,64]$ where the proton spectrum is expressed as:

$$
\frac{\mathrm{d} N}{\mathrm{~d} \varepsilon}=\frac{1.3 N_{\mathrm{Hot}} c_{\mathrm{s}}}{c \sqrt{2 \varepsilon T_{\mathrm{Hot}}}} e^{-\sqrt{\frac{2 \varepsilon}{T_{\mathrm{Hot}}}}} \quad \text { and } \quad c_{\mathrm{s}}=\sqrt{\frac{k_{B} T_{\mathrm{Hot}}}{m_{\mathrm{p}}}}
$$

Therefore the proton spectrum is adequate for extracting the electron temperature (which is time-integrated over the proton acceleration time $\tau_{\text {acc }}$. However, RCF data is needed to measure the total number of electrons which contribute to proton acceleration. An example of $T_{\mathrm{Hot}}=0.83 \mathrm{MeV}$ (electron sheath temperature) retrieved this way is shown in Fig. 13b. The 1D fit assumes a homogeneous electron population over the sheath.

It is useful to compare this deduced $T_{\text {Hot }}$ with results using other ways. A measurement using direct electron spectrometry performed with the same laser conditions and using the same target is shown in Fig. 13a. The spectrum exhibits two temperatures, (typical for such experiments conducted at the fundamental frequency and for which a preplasma exists at the target front surface). The very high temperature component could be associated with electrons accelerated in the large preplasma, at low numbers but high energies. The lower temperature component is $0.76 \mathrm{MeV}$ which is in relatively good agreement with the temperature deduced above from the proton spectrum.

A third technique that can be used to measure the electron temperature senses the plasma expansion induced by the hot electrons locally at the target rear surface [65]. Time and space resolved interferometry (TASRI) enables the retrieval of electron dynamics at the laser-solid interface with mean energies in the $\mathrm{MeV}$ range. The diagnostic detects the rear surface expansion of a solid target using a single chirped IR probe pulse that is reflected from the rear surface of the target. Its principle is illustrated in Fig. 14, in which strongly expanding regions of the target (e.g. on the laser axis) induce a strong phase shift in the probe pulse while the more slowly expanding regions (on the sides) induce little effect on the probe pulse. The pulse profile provides spatially resolved phase shift information. To get temporal resolution (as the phase evolves quickly in time), we use a chirped probe pulse where time is correlated to the instantaneous spectrum. The final CCD detector provides a 2D display of phase information where one axis is correlated to time (i.e. the spectrum) and the other axis is correlated to space. The spatial resolution is determined by characteristics of the optical system and is typically a few microns.

Using a probe pulse that was chirped linearly to about $30 \mathrm{ps}$ (FWHM), we obtain temporal resolution of $\sim 4$ ps [65]. Figure 14b plots the dephasing velocity (the temporal derivative of the measured phase) at three radii, $r=0$ (center of the irradiation zone by the probe pulse), 45 and $75 \mu \mathrm{m}$ that are taken from a phase map recorded at the rear target surface (the time origin corresponds to $\sim 1.6 \pm 1$ ps after the arrival of the peak of the main laser beam on target). Fitting the experimental dephasing velocities with those obtained numerically using an adiabatic expansion code [66], and two electron populations to drive the target expansion, one having a high mean energy but a low density (hot electrons) and the other of low mean energy at solid density (bulk electrons), we find that the dephasing velocities correspond to a hot electron density ranging from $4 \times 10^{19} \mathrm{~cm}^{-3}$ at $r=0$ to $2 \times 10^{19} \mathrm{~cm}^{-3}$ at the $75 \mu \mathrm{m}$ radius. The best fits for each radius have been obtained with a hot electron temperature of $0.85 \mathrm{MeV}$. Electrons are diluted within the target due to the ballistic spray which justifies the electron density changes in radial direction. The retrieved hot electron temperature is in good agreement with the values obtained above, either by fitting the proton spectrum or the direct measurement of the escaped electron spectrum. 

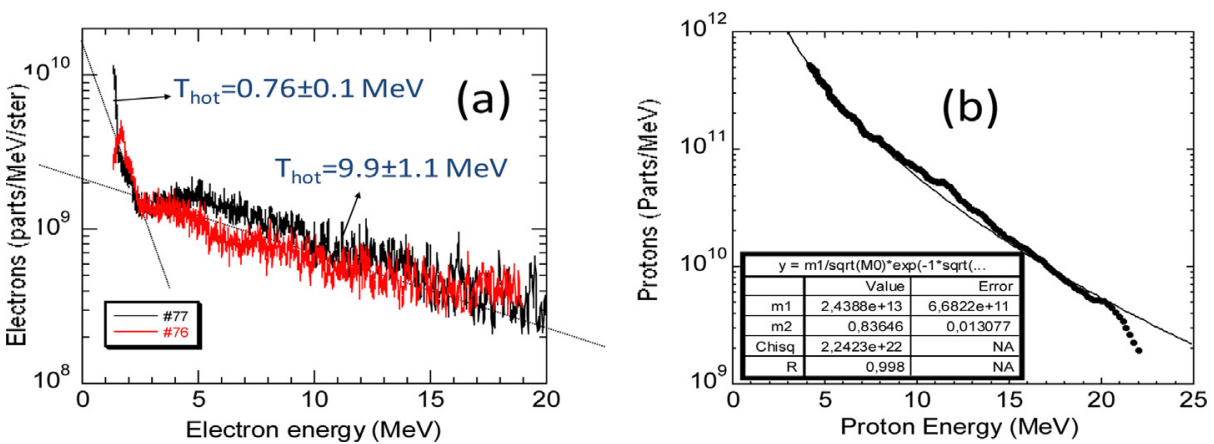

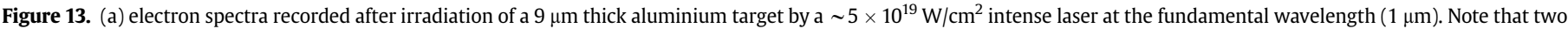
shots taken in the same conditions are overlaid. (b) Proton spectra obtained from the same two shots as in (a).

\section{Diagnostics using nuclear activation}

The acceleration of electrons, protons and heavier ions to tens of $\mathrm{MeV}$ energies in intense laser-matter interactions can induce nuclear reactions, both in the primary laser-irradiated target and in secondary activation samples. Thus nuclear activation can be used to diagnose properties of accelerated particle beams, as well as secondary energetic neutrons and Bremsstrahlung radiation. Protons and heavier ions with energies in the range of nuclear giant dipole resonances (a few to several tens of $\mathrm{MeV}$ ) can induce reactions such as proton-induced emission of nucleons. Higher energy protons, (of order hundreds of $\mathrm{MeV}$ ) can induce fission and spallation reactions. The low energy threshold for a given reaction is known as its activation energy.

Since the first experiments on proton and ion acceleration using short pulse high intensity lasers, nuclear activation diagnostics have been used to characterise the flux and spectral distributions of these beams [67-73]. This has generally involved well known reactions for proton-induced emission of nucleons or gamma radiation [67-70] and heavier ion-induced direct and compound nucleus reactions [70-73]. One or more well-known reactions are selected for measurement in a secondary sample positioned in the ion beam. A number of factors influence the reactions selected, including: (1) the activation energy; (2) knowledge of the cross section energy dependence; (3) the half-life of the decay product; and, (4) the availability of a sample with an appropriately high density of the stable parent nucleus.

Most of the selected reactions involve excitation of giant resonances which usually occur between a few and a few tens of MeV energy. The incident proton or heavier ion must be energetic enough to overcome the binding energy of the nucleon. The emission of a single neutron, for example, typically requires about 4-6 MeV. The most widely used reaction is the $(p, n)$ activation of
${ }^{63} \mathrm{Cu}$, which has a threshold energy of $4.15 \mathrm{MeV}$ (for proton energies above $60 \mathrm{MeV}$ other reaction cross sections also become significant). This results in the production of ${ }^{63} \mathrm{Zn}$, which decays by positron emission. The $511 \mathrm{keV}$ signature photons of positron annihilation are quantified using two NaI detectors operated in coincidence. High resolution Ge-crystal gamma-ray spectrometers are also used to measure signature gamma-rays from the radioactive decay of product nuclei. Thus the activity induced by protons within a given energy slice of the proton beam can be measured. By using stacked foils, the activation induced by protons over a wide range of energies can be determined. The spectral range covered depends on the stack thickness.

The number of reactions, $N$, induced by protons is:

$$
N=N_{0} \int_{E_{\text {thres }}}^{\infty} \sigma(E) n_{\mathrm{p}}(E) \mathrm{d} E
$$

where $N_{0}$ is the total number of irradiated nuclei in the secondary sample, $E_{\text {thres }}$ is the threshold energy of the reaction, $\sigma(E)$ is the energy dependant reaction cross section and $n_{\mathrm{p}}(E)$ is the incident proton energy distribution per unit area. By quantifying the number of reactions induced (considering the correction for decay time from the laser shot to the measurement), known $\sigma(E)$ and protonstopping data enable determination of the proton energy spectrum. The lower energy limit is the activation energy (i.e. $4.15 \mathrm{MeV}$ for $\left.{ }^{63} \mathrm{Cu}(\mathrm{p}, \mathrm{n})^{63} \mathrm{Zn}\right)$ and the upper limit is set by the number of induced reactions and the sensitivity of the detector.

Another technique uses a single foil and measurement of signature gamma rays from a range of decay products produced by multiple proton-induced reactions [68]. This may include the emission of different nucleons or multiple nucleons from the target
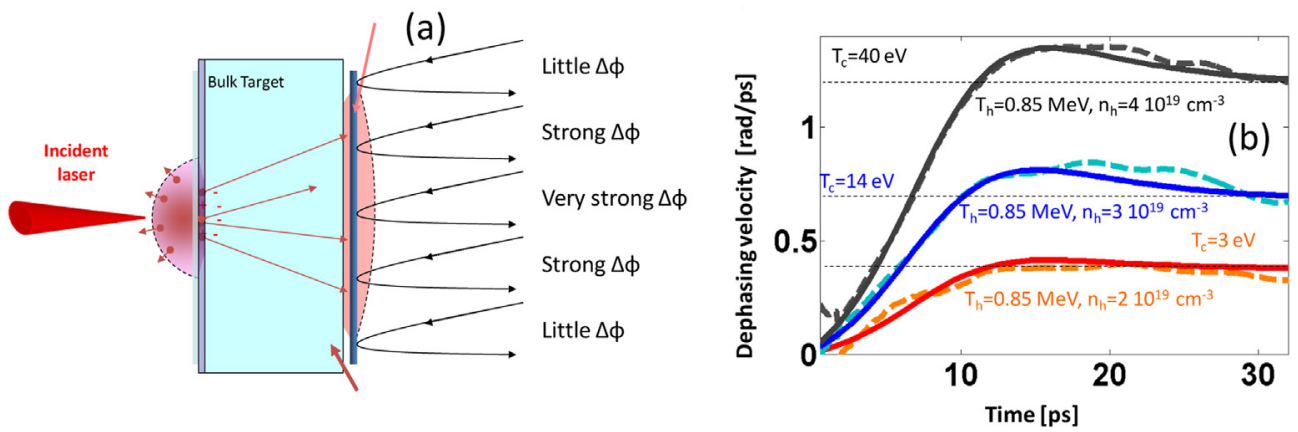

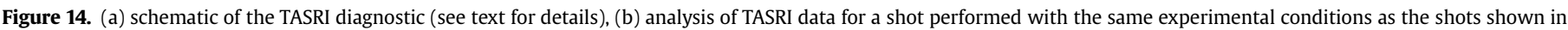
Fig. 16. 
nuclei. The use of the same reaction of different naturally occurring isotopes (e.g. ${ }^{65} \mathrm{Cu}$ and ${ }^{63} \mathrm{Cu}$ ) has also been demonstrated [68].

Furthermore, to characterise laser-produced particle and highenergy photon beams a nuclear pyrometry method based on nuclear activations has been developed [74]. The main component is a high-density target composed of several selected isotopes of different nuclear reaction thresholds. The decay spectrum of the activated target isotopes is measured using gamma spectroscopy. The use of the composite targets as well as an adequate analysis procedure which is described in Ref. [74] enables reconstruction of the initial particle spectra without anticipated fit procedures.

There is a wide variety of materials with isotope production cross-sections that can be used for this kind of beam monitoring. Examples include $\mathrm{Ti}$ and $\mathrm{Cd}$ as well as the $\mathrm{Cu}$ isotopes mentioned previously. The relevant details of some of these materials are shown in Table 2, some of which may become more relevant as the proton energies produced by laser sources increase. Studies have been made of the induced activation and the accuracy with which partial cross-sections are reproduced in radiation transport codes such as FLUKA [68]. Here it was found that the reactions with titanium and cadmium were modelled sufficiently well in FLUKA to produce accurate results of beam intensity for proton energies exceeding the reaction energy thresholds. For the $\mathrm{Cu}+\mathrm{p}$ reaction the results are in good agreement if the beam energy exceeds $\sim 14 \mathrm{MeV}$. Until now FLUKA was unable to correctly calculate the useful cross section for lower proton energies.

Activation techniques have also been developed to characterise the properties of heavier ion beams produced by the same laseracceleration mechanism [67,71,72]. Due to their much shorter range, heavier ions can stop in the first activation foil so the energy spectrum cannot easily be determined with a stacked foil arrangement. Instead multiple reaction channels in the same target foil have been used. The relevant reaction processes depend on the incident ion energy. However, ions will typically fuse with the nuclei in the secondary target, forming highly excited compound nuclei, which evaporate protons, neutrons and alpha particles [71]. A technique involving measurement of a range of different fusionevaporation channels was developed to measure the energy spectrum of ${ }^{56} \mathrm{Fe}$ ions in Ref. [67]. The spectrum extraction technique is similar to that described above for protons.

There are advantages to using a nuclear activation ion diagnostic. It can be used for single-shot, energy resolved measurements of ion flux over a large solid angle, i.e. the full beam. Thus the angularly resolved energy spectrum and intensity variations within the proton beam can be measured. Importantly, these measurements can be made over a very large dynamic range. Proton flux measurements have been made over 6 orders of magnitude, as demonstrated in Fig. 15 [70]. Thus activation of stacked foils can provide the same spectral and spatial intensity beam measurements as stacked dosimetry film (or CR39), over a dynamic range that is comparable or larger. The main drawback of a nuclear activation diagnostic is that the activated sample must typically be removed from the interaction chamber for off-line analysis. This

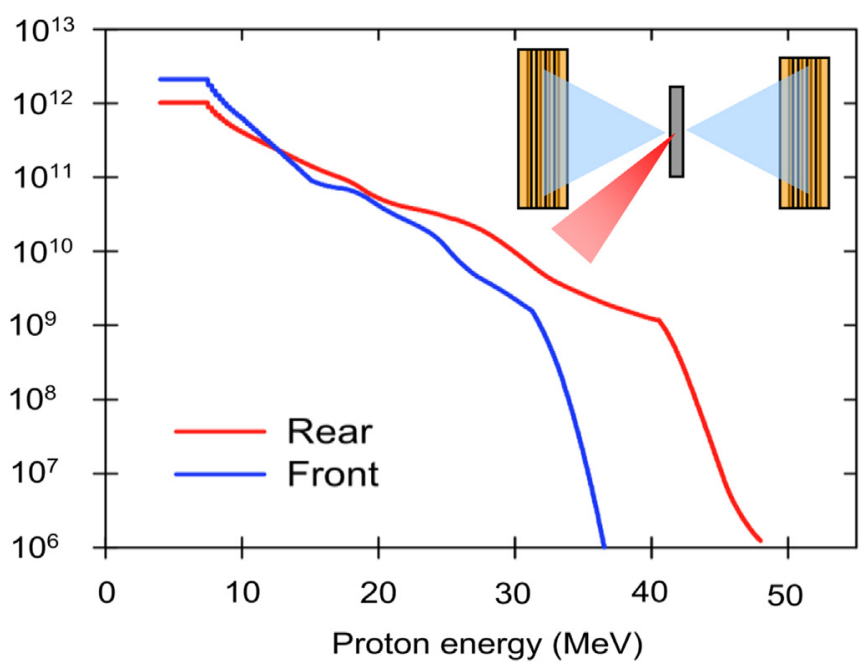

Figure 15. Example proton spectra, diagnosed using (p,n) reactions in copper, at the front and rear of a thin aluminium target foil irradiated by a high intensity picosecond laser pulse. Proton flux measurements spanning over six orders of magnitude are demonstrated. The arrangement is shown schematically in the inset.

involves special handling procedures for activated samples and typically long counting times. The use of nuclear detectors positioned inside the target vacuum chamber has been demonstrated [69] which extends the use of nuclear activation diagnostics to reactions with short-lived products.

Another development in nuclear diagnostics is the more sophisticated portable $32 \mathrm{NaI}$ detector integrated acquisition system named NATALIE (Nuclear Activation Technique for Analysis of Laser Induced Energetic particles) by F. Hannachi et al. [75]. This approach enables simultaneous measurements of the positronemission activation in each of the stacked copper foils, which speeds up the characterisation of laser produced energetic particle beams compared to the use of a single NaI coincidence counting detector system.

As discussed in Section 2, the technique of radiochromic film imaging spectroscopy (RIS) (see for example Ref. [8]) enables measurement of spatial and spectral profiles of the proton beam for a single laser shot. Although this technique is widely used, dosimetry film becomes saturated with high particle fluxes. Measurement of the spatial-intensity distribution of activated nuclei in secondary samples provides an alternative approach which does not saturate at the high fluxes where dosimetry film saturates. The nuclear activated imaging spectroscopy (NAIS) diagnostic is based on the nuclear activation of stacked copper foils. The reconstruction of the spectrally and spatially resolved beam profiles is performed via an autoradiography of activated copper foils using imaging plate (IP) detectors (see Fig. 16). The energy and spatial resolutions of the proton beam using NAIS are comparable to the resolution of the beam characterisation using the RIS technique.

Table 2

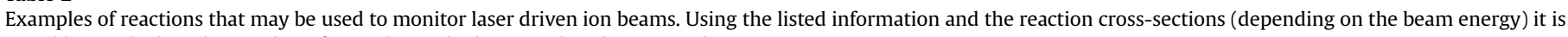
possible to calculate the number of particles in the beam and to determine their energy.

\begin{tabular}{|c|c|c|c|c|c|c|}
\hline $\begin{array}{l}\text { Initial } \\
\text { reaction }\end{array}$ & $\begin{array}{l}\text { First daughter } \\
\text { useful reaction }\end{array}$ & $\begin{array}{l}\text { Final daughter } \\
\text { useful reaction }\end{array}$ & $\begin{array}{l}\text { Energy } \\
\text { threshold }\end{array}$ & $\begin{array}{l}\text { Energy of the } \\
\text { useful gamma }\end{array}$ & $\begin{array}{l}\text { Half life of the } \\
\text { useful gamma }\end{array}$ & $\begin{array}{l}\text { Emission probability } \\
\text { of the useful gamma }\end{array}$ \\
\hline${ }^{63} \mathrm{Cu}+\mathrm{p}$ & ${ }^{63} \mathrm{Zn}+\mathrm{n}$ & ${ }^{63} \mathrm{Cu}+\gamma$ & $4.15 \mathrm{MeV}$ & $669 \mathrm{keV}$ & $38.47 \mathrm{~min}$ & $8 \%$ \\
\hline${ }^{110} \mathrm{Cd}+\mathrm{p}$ & ${ }^{109} \operatorname{In}+2 \mathrm{n}$ & ${ }^{109} \mathrm{Cd}+\gamma$ & $12.72 \mathrm{MeV}$ & $203 \mathrm{keV}$ & $4.17 \mathrm{~h}$ & $73.5 \%$ \\
\hline${ }^{48} \mathrm{Ti}+\mathrm{p}$ & ${ }^{44} \mathrm{Sc}+\mathrm{n}+\alpha$ & ${ }^{44} \mathrm{Ca}+\gamma$ & $13.88 \mathrm{MeV}$ & $1157 \mathrm{keV}$ & $3.97 \mathrm{~h}$ & $99.9 \%$ \\
\hline${ }^{63} \mathrm{Cu}+\alpha$ & ${ }^{66} \mathrm{Ga}+\mathrm{n}$ & ${ }^{66} \mathrm{Zn}+\gamma$ & $7.5 \mathrm{MeV}$ & $1039 \mathrm{keV}$ & $9.49 \mathrm{~h}$ & $36.9 \%$ \\
\hline
\end{tabular}




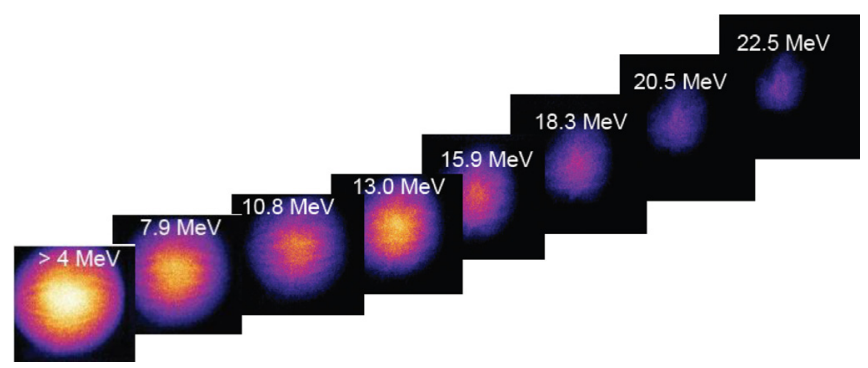

Figure 16. Autoradiography of activated copper foils using imaging plate (IP) detectors. The foils were activated by proton-induced nuclear reactions. The picture shows the beam profile at several proton energies. The proton beam was produced by a relativistic laser-matter interaction using a rear side micro-structured thin gold target. The autoradiography shows the imprint of the target micro-structure. This demonstrates the capability of NAIS to characterise laser accelerated proton beams.

\section{Targetry instrumentation for laser-accelerated ion sources}

\section{Unique features of targetry instrumentation}

The word "instrumentation" has specific meanings within 'Targetry' which are different from the meanings within Diagnostics. The first relevant meaning is equipment to "characterise" (or sometimes "metrologise") microtargets and their positioning. More precisely "characterisation" refers to the measurement of the physical properties of microtargets which can, for example, include chemical composition. The word "metrologise" is also used in this sense although preferably it is used to refer only to the physical form, of targets (shapes and sizes). Included within this sense is also equipment to measure target positioning (linear and angular) in an interaction environment. A second meaning of "instrumentation" relevant to Targetry is only recently becoming apparent which is equipment for monitoring a stream of targets delivered for extended periods in a high repetition-rate environment. This second sense is the same as that for high throughput manufacturing environments where (economically) efficient systems or plant operation is paramount, typically underpinned by optimisation considerations, such as lean six-sigma processes. Because there is not much experience with such systems being driven by high repetition-rate, high power lasers instrumentation challenges will need to be addressed when they come on line over the next few years.

Early experience at the Central Laser Facility of the Rutherford Appleton Laboratory fielding high repetition-rate experiments on the GEMINI laser [76] and work on the HiPER [77] and LIBRA projects [78] has given valuable practical insight into several important microtargetry issues. Some of the main observations are summarised in the following sections. Perhaps the most important lesson is the necessity to integrate microtarget production solutions with final microtarget positioning solutions.

\section{Microtarget production for repetition-rated operation}

Several techniques are well established for high repetition-rate targetry such as gas jets, tape drives and droplet generators. However, the techniques have (differing) limitations, most notably target geometry and complexity. Sophisticated 2D and 3D targets have been requested for high repetition-rate experiments. To understand the instrumentation required for microtarget characterisation it is appropriate to briefly outline some of the most common production techniques.

Thin and ultrathin foils can be coated onto substrates treated with releasing agents. Such foils can be floated off onto a water surface and subsequently lifted from the water onto a suitable mount, typically having through holes. Mounts enabling simultaneous mounting over an array (for example $5 \times 5$ ) of holes yielding multiple thin film targets have been developed. A challenge with these techniques is to ensure that the foils are sufficiently flat. Complex multilayer foil target arrays have been similarly prepared. Ultrathin foils (thinner than $50 \mathrm{~nm}$ ) in a range of materials (with the thinnest, although not in arrays, of $2.5 \mathrm{~nm}$ carbon) have also been produced [79].

Using ultra high precision micromachining like CNC milling it is possible to produce high aspect ratio 3D microparts with submicron accuracy. This technique has been developed at RAL to produce microparts (for example halfraums) in high yield batches.

A series of $2 \mathrm{D}$ and $21 / 2 \mathrm{D}$ microtargets have been fabricated at RAL using wafer-based MEMS techniques. A good example of 2D microtargets is the silicon nitride membrane targets used at RAL by Strangio et al., in 2006 [80] (see Fig. 17). Although initially expensive MEMS manufacturing does uniquely enable production of (large numbers of) microtargets or microcomponents. Additionally, if large numbers are required then significant cost savings have been demonstrated.

\section{High repetition rate microtarget placement in vacuum chamber}

High accuracy microtarget placement at high repetition-rates has become a significant technical challenge. Target wheels have been used in many facilities but have significant limitations if several tens of shots are required without breaking vacuum. In a joint collaboration between General Atomics and the CLF an Inserter system was developed to meet this requirement. The system comprises a linear arm equipped with grippers that can pick up targets mounted on specialised carriers and then place them on a hexapod (for accurate positioning) in the interaction chamber. Positional accuracies of a few microns can be achieved. Target carriers are individually identified using a machine readable (2D) bar code.

Devices like this allow multiple targets to be accurately positioned for shooting in a (near) continuous operation. Future projects, such as HiPER, will likely require development of high accuracy injector technology in which (cryogenic) microtargets will be injected and positioned in an interaction chamber without mechanical support.

\section{Established microtarget characterisation techniques and quality assurance}

Historically a complimentary range of characterisation equipment is used in Targetry and which has developed without the need

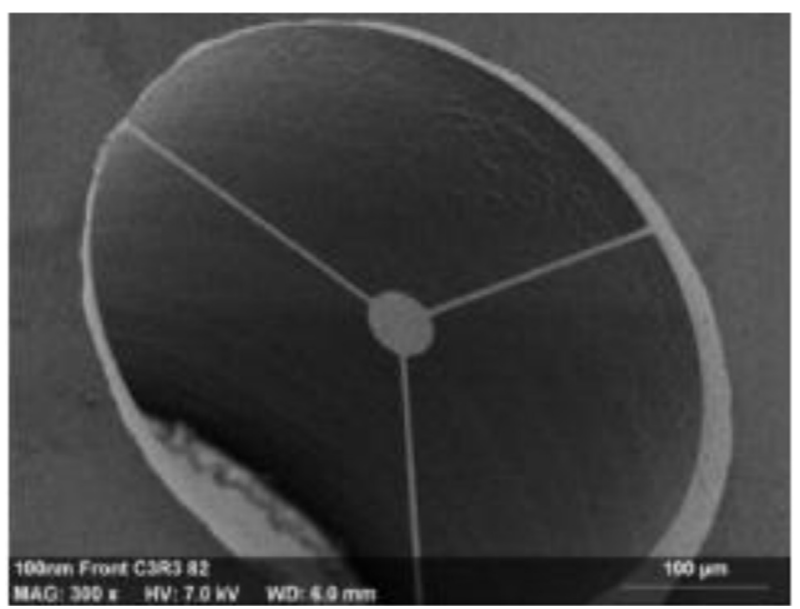

Figure 17. Microtarget example consisting of a 32 micron diameter, $40 \mathrm{~nm}$ thick silicon nitride membrane, fabricated at RAL using wafer-based MEMS techniques. 
for high throughput or rapid data collection. It is noteworthy that a large portion of characterisation time (easily over half) is spent during the development of production processes and for many, especially novel, target types relatively little time is required for quality assurance on finished product, not least due to a quality "gating" process throughout individual target production. Typical characterisation processes include; high resolution optical microscopy, tactile thin film measurement, scanning electron microscopy and white light interferometry. For more accurate measurements, for example sphere mapping, atomic force microscopy is used.

Several methods of automated metrology (amenable to repetition-rated operation) exist in various sectors of industry, for example automated CMMs (coordinate measuring machines). Some of the methods have been directly applied to mass-produced microtarget component metrology. Particular challenges have arisen for 3D microtargets and this may require the introduction of automation to techniques like confocal microscopy. One possibility is to store the information as a 3D spatial image (for example). This creates a large amount of metrology data (of the order of $1 \mathrm{~GB}$ per target) but there are significant advantages from reducing the amount of non-automated metrology and from introducing the possibility of post-hoc characterisation. A sophisticated data management system has been developed to record the characterisation data for each individual target and to synchronise it with individual shot data. Such high levels of individual target data control are highly amenable to quality management systems and several microtarget fabrication facilities have already introduced ISO9001, which would be an obvious international standard to use.

\section{High repetition rate logistics and methodology for targetry}

Target production and placement may become a limiting factor in future high repetition-rate experiments. Accurate insertion/injection of targets is a significant challenge and the solutions are intimately related to microtarget design and production. Also insertion mechanisms introduce further experimental complexity (and possibly extra characterisation). Due to microtarget production times (i) there can be a significant number of unshot targets following a high repetition rate experiment if it does not run smoothly and (ii) the ability to implement target modifications in response to ongoing experimental data is significantly reduced. Large amounts of metrology data are produced which needs careful control, especially in synchronising with other (shot and experimental diagnostic) data streams. To enable targetry activities new production and characterisation techniques will be required. There is a clear necessity for early and detailed planning of targetry.

\section{Conclusion}

We have described some novel and some established instrumentation techniques for laser-accelerated proton (ion) beams. Even with established diagnostics investigations of new details and comparisons continue. A good example is dynamic range enhancement with radiochromic film (RCF) afforded by multichannel (multi-colour) post-processing. Ideally, accelerator instrumentation that characterises and controls machine performance should be robust, based on a single pulse (and a single bunch) and noninvasive with prompt readout that provides adequate single bunch resolutions. Furthermore, for performance optimisation, it will be essential to uniquely correlate, for a single laser pulse and the specific particle bunch it generates, laser pulse, laser-plasma and particle bunch diagnostics at an operating repetition-rate. Novel instrumentation can address these ideal features. The use of inline multiple scintillators to promptly measure transverse beam profiles with energy resolution for a single bunch is a typical example. Another example is the demonstration of time and space-resolved interferometry (TASRI) which measures noninvasively the single shot details of electron dynamics to determine hot electron density and temperature at the target rear surface. These features can be uniquely correlated to properties of the driving laser pulse and the associated proton bunch.

Noteworthy are unique instrumentation requirements for targetry. Precise repetition-rated operation for solid targets remains a key issue for integrated laser-driven ion accelerator systems. We anticipate stringent requirements for rapid motion and precise positioning in addition to diagnostics to characterise the pre-irradiated target and the hosted laser-induced plasma. Integration of methodologies and metrologies for target production and micro-positioning is anticipated for ILDIAS development. In general, laser-acceleration of protons (ions) can bring unique bunch features (such as high peak current) and machine performance requirements (such as control of beam steering and bunch charge for a given energy). Continued advancement of new instrumentation technologies will be critical.

\section{Acknowledgements}

This work has been supported in part by Special Coordination Funds (SCF) for Promoting Science commissioned by the Ministry of Education, Culture, Sports, Science and Technology (MEXT) of Japan. Partial support is also provided by the DFG Cluster of Excellence: Munich-Centre for Advanced Photonics and the LIBRA EPSRC Basic technology grant.

\section{Figure permissions}

Figure 2 - Reprinted with the kind permission of the authors of [20] and Cambridge University Press.

Figure 10 - Reprinted with the kind permission of the authors of [47] and Elsevier.

Figure 13 - Reprinted with the kind permission of the authors of 'Antici P, Albertazzi B, Audebert P, Buffechoux S, Hannachi F, d'Humieres $\mathrm{E}$ et al. Measuring hot electron distributions in intense laser interaction with dense matter. New Journal of Physics 2012: 14; 063023. 10 pp.' and @IOP Publishing\& Deutsche Physicalische Geseilschaft CC BY-NC-SA.

Figure 14a - Reprinted with the kind permission of the authors of [65] and copyright (2010) AIP Publishing LLC.

Figure $14 \mathrm{~b}$ - Reprinted with the kind permission of the authors of [62] and copyright (2008) by the American Physical Society.

\section{References}

[1] Zeil K, Baumann M, Beyreuther E, Burris-Mog T, Cowan TE, Enghardt W, et al, Dose controlled irradiation of cancer cells with laser accelerated proton pulses. Appl Phys B 2013:110:437. 8pp.

[2] Fritzler S, Malka V, Grillon G, Rousseau JP, Burgy F, Lefebvre E, et al. Proton beams generated with high-intensity lasers: applications to medical isotope production. Appl Phys Lett 2003;83:3039. 3pp.

[3] Linz U, Alonso J. What will it take for laser driven proton accelerators to be applied to tumor therapy? Phys Rev ST Accel Beams 2007;10:094801. 8pp.

[4] Borghesi M, Campbell DH, Schiavi A, Haines MG, Willi O, MacKinnon AJ, et al Electric field detection in laser-plasma interaction experiments via the proton imaging technique. Phys Plasmas 2002;9:2214. 7pp.

[5] Bartal T, Foord ME, Bellei C, Key MH, Flippo KA, Gaillard SA, et al. Focusing of short-pulse high-intensity laser accelerated proton beams. Nat Phys 2012;8: 139. 4pp.

[6] Antici P, Migliorati M, Montacci A, Picardi L, Palumbo L, Rousivalle C. A compact post-acceleration scheme for laser-generated protons. Phys Plasmas 2011:18:073103. 11pp.

[7] Technical Report Series No. 398, IAEA 2000. Absorbed dose determination in external beam radiotherapy. http://www-pub.iaea.org/mtcd/publications/pdf/ trs398_scr.pdf. 
[8] Nürnberg F, Schollmeier M, Brambrink E, Blaževic A, Carroll D, Flippo K, et al Radiochromic film imaging spectroscopy of laser-accelerated proton beams. Rev Sci Instrum 2009;80:033301. 13pp.

[9] Hey DS, Key MH, Mackinnon AJ, MacPhee AG, Patel PK, Freeman RR. Use of GafChromic film to diagnose laser generated proton beams. Rev Sci Instrum 2008;79:053501. 4pp.

[10] Niroomand-Rad A, Blackwell CR, Coursey BM, Gall KP, Galvin JM, McLaughlin WL, et al. Radiochromic film dosimetry: recommendations of AAPM Radiation Therapy Committee Task Group 55. Med Phys 1998;25:2093. 23pp.

[11] Alva H, Mercado-Uribe H, Rodriguez-Villafuerte M, Brandan ME. The use of a reflective scanner to study radiochromic film response. Phys Med Biol 2002; 47:2925. 9pp.

[12] Martišíková M, Jäkel O. Dosimetric properties of Gafchromic EBT films in monoenergetic medical ion beams. Phys Med Biol 2010;55:3741. 11pp.

[13] Butson MJ, Yu PKN, Cheung T, Metcalfe P. Radiochromic film for medical radiation dosimetry. Mater Sci Eng 2003;41:61. 59pp.

[14] Cowan JS, Flippo KA, Gaillard SA. Characterization of radiochromic film scanning techniques used in short-pulse-laser ion acceleration. Rev Sci Instrum 2008;79:10E535. 5pp.

[15] GafChromic radiochromic film types HD-810, HS, MD-55. Wayne, New Jersey 07470, USA: ISP Corporation; 2008http://www.ispcorp.com/products/dosimetry/.

[16] Cowan T, Fuchs J, Ruhl H, Kemp A, Audebert P, Roth M, et al. Ultralow emittance, multi-MeV proton beams from a laser virtual-cathode plasma accelerator. Phys Rev Lett 2004;92:204801. 4pp.

[17] Ruhl H, Cowan TE, Fuchs J. The generation of micro-fiducials in laser-accelerated proton flows, their imaging property of surface structures and application for the characterization of the flow. Phys Plasmas 2004;11:L17. 4pp.

[18] Roth M, Blaževic A, Geissel M, Schlegel T, Cowan TE, Allen M, et al. Energetic ions generated by laser pulses: a detailed study on target properties. Phys Rev ST Accel Beams 2002:5:061301. 8pp.

[19] Breschi E, Borghesi M, Galimberti M, Giulietti D, Gizzi LA, Romagnani L. A new algorithm for spectral and spatial reconstruction of proton beams from dosimetric measurements. Nucl Instrum Methods Phys Res A 2004;522:190. 6pp.

[20] Kirby D, Green S, Fiorini F, Parker D, Romagnani L, Doria D, et al. Radiochromic film spectroscopy of laser-accelerated proton beams using the FLUKA code and dosimetry traceable to primary standards. Lasers Part Beams 2011;29:231. 9pp.

[21] Ziegler JF. SRIM-2003. Nucl Instrum Methods Phys Res B 2004;219:1027. 10pp.

[22] Devic S, Tomic N, Soares CG, Podgorsak EB. Optimizing the DR extension of a radiochromic film dosimetry system. Med Phys 2009;36:429. 9pp.

[23] Piermattei A, Miceli R, Azario L, Fidanzio A, delle Canne S, De Angelis C, et al. Radiochromic film dosimetry of a low energy proton beam. Med Phys 2000;27:1655. 6pp.

[24] Vatnitsky SM. Radiochromic film dosimetry for clinical proton beams. Appl Radiat Isot 1997;48:643. 9pp.

[25] Daftari I, Castenadas C, Petti PL, Singh RP, Verhey LJ. An application of GafChromic MD-55 film for $67.5 \mathrm{MeV}$ clinical proton beam dosimetry. Phys Med Biol 1999;44:2735. 11pp.

[26] Kojima T, Sunaga H, Takizawa H, Hanaya H, Tachibana H. 3-45 MeV/u ion beam dosimetry using thin film dosimeters. Radiat Phys Chem 2003;68:975. 6pp.

[27] Kirby D, Green S, Palmans H, Hugtenburg R, Wojnecki C, Parker D. LET dependence of GafChromic films and an ion chamber in low-energy proton dosimetry. Phys Med Biol 2010;55:417. 17pp.

[28] http://www.fujifilm.com/products/life_science/index_en.html.

[29] Paterson IJ, Clarke RJ, Woolsey NC, Gregori G. Image plate response for conditions relevant to laser-plasma interaction experiments. Meas Sci Technol 2008;19:095301. 5pp.

[30] Miyahara J, Takahashi K, Amemiya Y, Kamiya N, Satow Y. A new type of X-ray area detector utilizing laser stimulated luminescence. Nucl Instrum Methods Phys Res A 1986;246:572. 7pp.

[31] Amemiya Y, Matsushita T, Nakagawa A, Satow Y, Miyahara J, Chikawa J. Design and performance of an imaging plate system for X-ray diffraction study. Nucl Instrum Methods Phys Res A 1988;266:645. 9pp.

[32] Cookson DJ. Calculation of absolute intensities from x-ray imaging plates. J Synchrotron Radiat 1998:5:1375. 8pp.

[33] Inoue K, Oka T, Miura K, Yagi N. Present status of BL40B2 and BL40XU at Spring-8 (beamlines for small angle X-ray scattering). In: American Institute of Physics Conference series, vol. 705; 2004. p. 336. 4pp.

[34] Niimura N, Karasawa Y, Tanaka I, Miyahara J, Takahashi K, Saito H, et al. An imaging plate neutron detector. Nucl Instrum Methods Phys Res A 1994;349: 521. $5 \mathrm{pp}$.

[35] Schlapp M, Conrad H, von Seggern H. Pixelated neutron image plates. J Phys D Appl Phys 2004;37:2607. 6pp.

[36] Mančić A, Fuchs J, Antici P, Gaillard SA, Audebert P. Absolute calibration of photostimulable image plate detectors used as (0.5-20 MeV) high-energy proton detectors. Rev Sci Instrum 2008;79:073301. 6pp.

[37] Doria D, Kar S, Kakolee K, Ramakrishna B, Sarri G, Quinn KE, et al. Calibration of image plate response to energetic carbon ions. Central Laser Facility Annual Report 2009/2010. p.46. 1pp.

[38] Gaillard S, Fuchs J, Renard-LaGalloudec N, Cowan TE. Study of saturation of CR-39 nuclear track detectors at high ion fluence and of associated artifact patterns. Rev Sci Instrum 2007;78:013304. 13pp.

[39] Metzkes J, Karsch L, Kraft S, Pawelke J, Richter C, Schurer M, et al. A scintillator-based online detector for the angularly resolved measurement of laser-accelerated proton spectra. Rev Sci Instrum 2012;83:123301. 7pp.
[40] Jakubek J. Semiconductor pixel detectors and their applications in life sciences. J Instrum 2008;4:P0301. 22pp.

[41] Knoll GF. Radiation detection and measurements I. John Wiley \& Sons; 1989.

[42] Wilson P, Jones B, Yokoi T, Hill M, Vojnovic B. Revisiting the ultra-high dose rate effect: implications for charged particle radiotherapy using protons and light ions. Br J Radiol 2012;85:e933. 7pp.

[43] Reinhardt S. Detection of laser-accelerated protons. München: Ludwig-Maximilians Universität München; 2012.

[44] Fletcher KA, Apker B, Hammond S, Punaro J, Marshall FJ, Laine J, et al. Detection of charged particles with charge injection devices. Rev Sci Instrum 2007;78:063301. 4pp.

[45] Burke B, Jorden P, Vu P. CCD technology. Exp Astron 2005;19:69. 34pp. [compact review from MIT/Lincoln Labs., e2v technology and Fairchild Imaging]

[46] http://sales.hamamatsu.com/en/products/electron-tube-division/detectors/ microchannel-plates-mcps.php.

[47] Prasad R, Doria D, Ter-Avetisyan S, Foster PS, Quinn KE, Romagnani L, et al. Calibration of Thomson parabola-MCP assembly for multi-MeV ion spectroscopy. Nucl Instrum Methods Phys Res A 2010;623:712. 4pp.

[48] Thomson JJ. Rays of positive electricity. Phil Mag 1911;21:225. 25pp.

[49] Sisoev AA, Schupachin MC. Introduction to mass-spectroscopy. Moscow: Atomizdat; 1977.

[50] Schreiber J, Ter-Avetisyan S, Risse E, Kalachnikov MP, Nickles PV, Sandner W, et al. Pointing of laser-accelerated proton beams. Phys Plasmas 2006;13: 033111. 5pp.

[51] Ter-Avetisyan S, Schnürer M, Busch S, Risse E, Nickles PV, Sandner W. Spectral dips in ion emission emerging from ultrashort laser-driven plasmas. Phys Rev Lett 2004;93:155006. 4pp.

[52] Ter-Avetisyan S, Schnürer M, Nickles PV, Sikollik T, Risse E, Kalashnikov M, et al. The Thomson deflectometer: a novel use of the Thomson spectrometer as a transient field and plasma diagnostic. Rev Sci Instrum 2008;79:033303. 5pp.

[53] Sokollik T, Schnurer M, Ter-Avetisyan S, Nickles PV, Risse E, Kalashnikov M, et al. Transient electric fields in laser plasmas observed by proton streak deflectometry. Appl Phys Lett 2008;92:091503. 3pp.

[54] Ter-Avetisyan S, Schnurer M, Nickles PV, Sandner W, Nakamura T, Mima K. Correlation of spectral, spatial and angular characteristics of an ultrashort laser driven proton source. Phys Plasmas 2009;16:043108. 6pp.

[55] Chen H, Hazi AU, van Maren R, Chen SN, Fuchs J, Gauthier M, et al. An imaging proton spectrometer for short-pulse laser plasma experiments. Rev Sci Instrum 2010;81:10D314. 4pp.

[56] Harres K, Schollmeier M, Brambrink E, Audebert P, Blazevic A, Flippo K, et al. Development and calibration of a Thomson parabola with microchannel plate for the detection of laser-accelerated MeV ions. Rev Sci Instrum 2008;79: 093306. 9pp.

[57] Ter-Avetisyan S, Ramakrishna B, Doria D, Sarri G, Zepf M, Borghesi M, et al. Complementary ion and ultra-violet spectrometer for laser-plasma diagnosis. Rev Sci Instrum 2009;80:103302. 5pp.

[58] Roth M, Brambrink E, Audebert P, Basko M, Blazevic A, Clarke R, et al. Laseraccelerated ions in ICF research prospects and experiments. Plasma Phys Control Fusion 2005;47:B841. 10pp.

[59] Mancic A, Robiche J, Antici P, Audebert P, Blancard C, Combis P, et al. Isochoric heating of solids by laser-accelerated protons: experimental characterization and self-consistent hydrodynamic modeling. High Energy Density Phys 2010;6:21. 8pp.

[60] Borghesi M, Fuchs J, Bulanov SV, Mackinnon AJ, Patel PK, Roth M. Fast ion generation by high-intensity laser irradiation of solid targets and applications. Fusion Sci Technol 2006;49:412. 28pp.

[61] Brambrink E, Roth M, Blazevic A, Schlegel T. Modeling of the electrostatic sheath shape on the rear target surface in short-pulse laser-driven proton acceleration. Lasers Part Beams 2006;24:163. 6pp.

[62] Antici P, Fuchs J, Borghesi M, Gremillet L, Grismayer T, Sentoku Y, et al. Hot and cold electron dynamics following high-intensity laser-matter interactions. Phys Rev Lett 2008:101:105004. 4pp.

[63] Mora P. Plasma expansion into a vacuum. Phys Rev Lett 2003;90:185002. 4pp.

[64] Fuchs J, Antici P, d'Humieres E, Lefebvre E, Borghesi M, Brambrink E, et al. Laser-driven proton scaling laws and new paths towards energy increase. Nat Phys 2006;2:48. 7pp.

[65] Antici P, Chen SN, Gremillet L, Grismayer T, Mora P, Audebert P, et al. Time and space resolved interferometry for laser-generated fast electron measurements. Rev Sci Instrum 2010:81:113302. 10pp.

[66] Mora P. Thin-foil expansion into a vacuum. Phys Rev E 2005;72:056401. 5pp.

[67] Spencer I, Ledingham KWD, Singal RP, McCanny T, McKenna P, Clark E, et al. Laser generation of proton beams for the production of short-lived positron emitting radioisotopes. Nucl Instrum Methods Phys Res B 2001;183:449. 10pp.

[68] Yang JM, McKenna P, Ledingham KWD, McCanny T, Shimizu S, Robson L, et al. Nuclear reactions in copper induced by protons from a petawatt laser-foil interaction. Appl Phys Lett 2004;84:675. 3pp.

[69] Clarke RJ, Ledingham KWD, McKenna P, Robson L, McCanny T, Neely D, et al. Detection of short lived radioisotopes as a fast diagnostic for intense lasersolid interactions. Appl Phys Lett 2006;89:141117. 3pp.

[70] McKenna P, Ledingham KWD, Yang JM, Robson L, McCanny T, Shimizu S, et al. Characterization of proton and heavier ion acceleration in ultrahigh-intensity laser interactions with heated target foils. Phys Rev E 2004;70:036405. 6pp.

[71] McKenna P, Ledingham KWD, McCanny T, Singhal RP, Spencer I, Santala MIK, et al. Demonstration of fusion-evaporation and direct-interaction nuclear 
reactions using high-intensity laser-plasma-accelerated ion beams. Phys Rev Lett 2003;91:075006. 4pp.

[72] McKenna P, Ledingham KWD, McCanny T, Singhal RP, Spencer I, Clark EL, et al. Effect of target heating on ion-induced reactions in high-intensity laserplasma interactions. Appl Phys Lett 2003;83:2763. 3pp.

[73] Offermann DT, Flippo KA, Cobble J, Schmitt MJ, Gaillard SA, Bartal T, et al. Characterization and focusing of light ion beams generated by ultra-intensely irradiated thin foils at the kilojoule scale. Phys Plasmas 2011;18:056713. 7pp.

[74] Günther MM, Sonnabend K, Brambrink E, Vogt K, Bagnoud V, Harres K, et al. A novel nuclear pyrometry for the characterization of high-energy Bremsstrahlung and electrons produced in relativistic laser-plasma interactions. Phys Plasmas 2011;18:083102. 8pp.
[75] Tarisien M, Plaisir C, Gobet F, Hannachi F, Aleonard MM, Rebii A. NATALIE: a 32 detector integrated acquisition system to characterize laser produced energetic particles with nuclear techniques. Rev Sci Instrum 2011;82:023302. 6pp.

[76] http://www.clf.rl.ac.uk/Facilities/Astra/Astra+Gemini/12258.aspx.

[77] http://www.qub.ac.uk/sites/LIBRA/.

78] http://www.hiper-laser.org/.

[79] Levin J, Knoll L, Scheffel M, Schwalm D, Wester R, Wolf A, et al. Application of ultrathin diamond-like-carbon targets to Coulomb explosion imaging. Nucl Instrum Methods Phys Res B 2000;168:268. 8pp.

[80] Strangio C, Caruso A, Neely D, Andreoli PL, Anzalone R, Clarke R, et al. Production of multi-MeV per nucleon ions in the controlled amount of matter mode (CAM) by using causally isolated targets. Lasers Part Beams 2007;25:85. 7pp. 